\title{
Integral modular data and congruences
}

\author{
Michael Cuntz
}

Received: 8 November 2006 / Accepted: 22 April 2008 / Published online: 13 May 2008

(C) Springer Science+Business Media, LLC 2008

\begin{abstract}
We compute all fusion algebras with symmetric rational $S$-matrix up to dimension 12. Only two of them may be used as $S$-matrices in a modular datum: the $S$-matrices of the quantum doubles of $\mathbb{Z} / 2 \mathbb{Z}$ and $S_{3}$. Almost all of them satisfy a certain congruence which has some interesting implications, for example for their degrees. We also give explicitly an infinite sequence of modular data with rational $S$ - and $T$-matrices which are neither tensor products of smaller modular data nor $S$-matrices of quantum doubles of finite groups. For some sequences of finite groups (certain subdirect products of $S_{3}, D_{4}, Q_{8}, S_{4}$ ), we prove the rationality of the $S$-matrices of their quantum doubles.
\end{abstract}

Keywords Modular data · Fusion algebra - Quantum double · Fourier matrix · Modular group

\section{Introduction}

The modular datum is a structure which appears in physics as well as in representation theory. It mainly consists of complex matrices $S$ and $T$ defining a representation of the modular group $\mathrm{SL}_{2}(\mathbb{Z})$ where the matrix $S$ may be used to define structure constants of a fusion algebra via the formula of Verlinde. There is almost nothing known about how to classify modular data, it is even unknown if there exist infinitely many modular data in some dimension.

The fusion algebras associated to some special cases have been investigated thoroughly, for example the affine data coming from Kac-Moody algebras have completely been classified by Gannon in [5]. Another important class of modular data is

M. Cuntz ( $\varangle)$

Universität Kaiserslautern, Postfach 3049, 67653 Kaiserslautern, Germany

e-mail: cuntz@mathematik.uni-kl.de 
given by the quantum doubles of finite groups (an overview may be found in [2]) and there is also a version twisted by a 3-cocycle (see [4]).

A very interesting connection is the one to the representation theory of finite groups of Lie type (see [9]). Also, there is a new theory in which a new structure called 'Spetses' is associated to a complex reflection group in a similar way as in Lusztig's theory (see [12]). There exist modular data for the Spetses (see for example [11] for the imprimitive case). Some of the corresponding fusion algebras have negative structure constants. The analogous constructions for finite Coxeter groups also yield Fourier matrices, but they are not always symmetric (see [8]). In fact, this shows that there are many structures which are almost modular data in the sense of Gannon [6] and therefore there is no 'correct' definition for a modular datum.

Here, we try to approach a classification of a restricted case, namely the case in which the matrix $S$ is rational. The number of such matrices $S$ in each dimension is then finite, so we can compute all of them up to a given dimension. It is very surprising that there are only two rational $S$-matrices up to dimension 12 (up to permutations of columns): the $S$-matrices of the quantum doubles of $\mathbb{Z} / 2 \mathbb{Z}$ and $S_{3}$.

The symmetric rational matrices $S$ in dimension up to 12 which define fusion algebras but do not necessarily have an associated $T$-matrix enjoy some very interesting properties. One of them is a certain congruence property which we try to understand in more details. We call such matrices congruence multiplication tables. We prove some relations between their degrees and that they exist in all dimensions except $2,3,5$ and 6 .

We also give an infinite sequence of modular data with rational $S$ - and $T$-matrices. The smallest new case in this sequence has dimension 94 and is neither a tensor product of smaller modular data nor an $S$-matrix of a quantum double of a finite group (even its fusion algebra does not come from a quantum double). This shows how far away we are from a classification: there are still examples which do not appear in the list of examples given by Gannon in [6].

In the next section, we give some infinite sequences of finite group modular data which are 'integral' and not just tensor products of smaller data. The last section is a collection of open questions.

The appendix contains a complete list of all symmetric rational Fourier matrices (see Definition 3.1) up to dimension 12 and a short explanation how it has been computed.

In dimension 12 there is a congruence multiplication table which has a $T$-matrix of infinite order. It has negative structure constants. In dimension 16, there is also a congruence multiplication table which is not the $S$-matrix of a quantum double and has a $T$-matrix of infinite order. It has nonnegative structure constants.

In dimension 18 there exists a modular datum with rational $S$-matrix. It is the smallest datum known to the author whose fusion algebra is not isomorphic to a fusion algebra coming from a quantum double, which is rational, has positive structure constants and for which the group $\langle S, T\rangle$ is finite. Up to 4-th roots of unity, this is the datum belonging to the 5-th family (in the numbering of the package CHEVIE, [7]) of the exceptional complex reflection group $G_{8}$ (the 8-th group in the Shephard-Todd numbering (see [13])). 


\section{Modular data}

Modular data are structures being studied in a large variety of contexts. The most common definition is the following (compare [6]):

Definition 2.1 Let $n \in \mathbb{N}$. By a modular datum we mean a pair $S, T \in \mathbb{C}^{n \times n}$ of matrices such that:

(1) $S$ is unitary and symmetric: $S \bar{S}^{T}=1, S=S^{T}$,

(2) $T$ is diagonal and of finite order,

(3) $S_{i 1}>0$ for $1 \leq i \leq n$,

(4) $S^{2}=(S T)^{3}$,

(5) $N_{i j}^{l}:=\sum_{k} \frac{S_{k i} S_{k j} \overline{S_{k l}}}{S_{k 1}} \in \mathbb{Z}$ for all $1 \leq i, j, l \leq n$.

Because of (1), (4) and (5), the matrices $S$ and $T$ define a representation of the modular group $\mathrm{SL}_{2}(\mathbb{Z})$, which is the reason why the datum above is called modular (see for example [6], 4 for a proof).

Definition 2.2 We will call a matrix $S$ satisfying the axioms (1), (3) and (5) a Fourier matrix as in [10].

The numbers $N_{i j}^{l}$ are called structure constants and define an associative commutative $\mathbb{Z}$-algebra which is a free $\mathbb{Z}$-module of finite rank (see for example [3], 2.1.3). Algebras coming from a Fourier matrix or a character table via such structure constants are usually called table algebras or fusion algebras (complex conjugation on the set of columns corresponds to an involution with certain properties). Remark that we do not assume that the structure constants $N_{i j}^{l}$ are nonnegative, because we would exclude many interesting examples. This is not so usual.

Definition 2.3 We call the algebra defined by the structure constants of a Fourier matrix a fusion algebra.

Gannon ([6]) gives a list of open questions involving modular data. The most important one is of course a classification. However this is probably a very difficult task because there are even much more fusion algebras than the long list of examples given by Gannon in [6]. It is also unknown, whether there exists a dimension $n$ for which there are infinitely many modular data. Most likely there are some infinite sequences that remain to be found.

\section{Integral modular data}

\subsection{Definition}

We would like to approach the first of these problems by restricting to certain modular data. The numbers $S_{i j} / S_{i 1}$ of an arbitrary modular datum are eigenvalues of matrices 
$N_{i}$ with integer entries (see Remark 3.2) and are therefore algebraic integers. So in particular if $S$ has only rational entries, then the matrix $s=\left(s_{i j}\right)_{i, j}$ defined by

$$
s_{i j}:=\frac{S_{i j}}{S_{i 1}}
$$

lies in $\mathbb{Z}^{n \times n}$. This leads us to the following definition:

Definition 3.1 Let $n \in \mathbb{N}$. An integral modular datum consists of matrices $s \in$ $\mathbb{Z}^{n \times n}$ and $T \in \mathbb{C}^{n \times n}$ such that:

(1) $s_{i 1}=1$ for $1 \leq i \leq n, \operatorname{det}(s) \neq 0$,

(2) $s s^{T}=\operatorname{diag}\left(d_{1}, \ldots, d_{n}\right)=: d$, so $d_{i}=\sum_{j} s_{j i}^{2}$,

(3) $\sqrt{d_{j}} s_{i j}=\sqrt{d_{i}} s_{j i}$ for $1 \leq i, j \leq n$ ( $s$ is symmetrizable),

(4) $N_{i j}^{l}:=\sum_{k} \frac{s_{k i} s_{k j} s_{k l}}{d_{k}} \in \mathbb{Z}$ for $1 \leq i, l \leq n$,

(5) $T$ is diagonal,

(6) $S^{2}=(S T)^{3}$ where $S_{i j}:=s_{i j} / \sqrt{d_{i}}$.

Here, $\sqrt{d_{i}}$ is chosen greater than 0 . The matrix $s$ is the integral Fourier matrix of the datum.

The simplest example is the datum where $s$ and $T$ are both the $1 \times 1$ matrix $s=$ $T=(1)$. This example is so trivial that it will be ignored in the remainder of the paper.

It does not really make a difference if we use $s$ instead of $S$. The matrix $s$ has the advantage, that it has only integer entries, but it has the disadvantage that it is not symmetric. Remark also that we do not require that $T$ has finite order. This is because there exist some interesting examples which satisfy all axioms except the finiteness of the group $\langle S, T\rangle$.

So a modular datum $(S, T)$ with $S \in \mathbb{Q}^{n \times n}$ is integral, and of course, integral modular data with $T$ of finite order are modular data. But there exist also integral modular data for which the matrix $S$ has irrational entries:

Example 1 Let $\zeta \in \mathbb{C}$ be a primitive 24-th root of unity. Then

$$
S:=\frac{1}{\sqrt{2}}\left(\begin{array}{cc}
1 & 1 \\
1 & -1
\end{array}\right), \quad s:=\left(\begin{array}{cc}
1 & 1 \\
1 & -1
\end{array}\right), \quad T:=\left(\begin{array}{cc}
\zeta & 0 \\
0 & -\zeta^{7}
\end{array}\right),
$$

define an integral modular datum.

Remark 3.2 If the structure constants of $s$ are nonnegative, then the theorem of Perron-Frobenius on nonnegative matrices applies to the matrices $N_{i}:=\left(N_{i j}^{k}\right)_{j, k}$ for $1 \leq i \leq n$ : if $s_{k}$ is the $k$-th row vector of $s$, then

$$
s_{k i} s_{k}^{T}=N_{i} s_{k}^{T}
$$

because the rows of $s$ are the one dimensional representations of the fusion algebra. So $s_{k}^{T}$ is an eigenvector of $N_{i}$ to the eigenvalue $s_{k i}$. The theorem says that there exists 
an eigenvalue of maximal absolute value and that it has an eigenvector with positive real coordinates. Since $s_{1}$ is the only row with positive coordinates (because the rows are orthogonal), this gives us the bound

$$
\left|s_{i j}\right| \leq s_{1 j}
$$

for all $i, j$. Conversely, it does not follow from this bound that the structure constants are nonnegative: There exist Fourier matrices $s$ with negative structure constants satisfying this bound.

Some well known examples for integral modular data are given by the quantum double of a finite group. The category of representations of this algebra is a modular tensor category (see for example [1], 3.2). For example for the groups $C_{2}, S_{3}, S_{4}, D_{4}$ we get indeed matrices $S$ with rational entries.

For given modular data $\left(S_{1}, T_{1}\right)$ and $\left(S_{2}, T_{2}\right)$, the tensor product matrices $S_{1} \otimes S_{2}$, $T_{1} \otimes T_{2}$ form again a modular datum. So from now on, we will only consider matrices which do not decompose as tensor products. Note however that a decomposition in a tensor product of matrices which may not be decomposed further is not unique, see the example below.

Definition 3.3 We will call a modular datum indecomposable if it may not be written as the tensor product of two smaller modular data.

Also, we will consider modular data only up to a simultaneous permutation of rows and columns fixing the 1 . Remark that for the fusion algebra, it makes no difference if you permute rows or columns (independently).

Definition 3.4 Let $(s, T),\left(s^{\prime}, T^{\prime}\right)$ be integral modular data of dimension $n$. Then $(s, T)$ and $\left(s^{\prime}, T^{\prime}\right)$ are called isomorphic if there exists a permutation $\sigma \in S_{n}$ such that

$$
s_{i j}=s_{\sigma(i) \sigma(j)}^{\prime}, \quad T_{i j}=T_{\sigma(i) \sigma(j)}^{\prime} \quad \text { for all } 1 \leq i, j \leq n .
$$

In the following lemmas, $(s, T)$ is an integral modular datum and

$$
s s^{T}=\operatorname{diag}\left(d_{1}, \ldots, d_{n}\right)=: d .
$$

Lemma 3.5 For a given dimension n, there is only a finite number of integral Fourier matrices.

Proof The symmetry of $S$ and 3.1, (1) give

$$
\frac{\sqrt{d_{1}}}{\sqrt{d_{i}}}=\frac{\sqrt{d_{1}}}{\sqrt{d_{i}}} s_{i 1}=s_{1 i},
$$

so $d_{1}=\sum_{i} s_{1 i}^{2}=\sum_{i} \frac{d_{1}}{d_{i}}$, or

$$
\frac{1}{d_{1}}+\cdots+\frac{1}{d_{n}}=1
$$


which has a finite number of solutions $\left(d_{1}, \ldots, d_{n}\right) \in \mathbb{Z}_{>0}^{n}$. The $i$-th row vector of $s$ has integer entries and length $\sqrt{d_{i}}$. So the number of integral Fourier matrices with given $d_{1}, \ldots, d_{n}$ is finite.

In fact, this shows even more: since $\frac{\sqrt{d_{1}}}{\sqrt{d_{i}}}=s_{1 i} \in \mathbb{Z}, d_{i}$ is a divisor of $d_{1}$ and $\frac{d_{1}}{d_{i}} \in \mathbb{Z}$ is a square for all $1 \leq i \leq n$. Also, $\frac{d_{i}}{d_{j}}$ is a square in $\mathbb{Q}$ for all $1 \leq i, j \leq n$.

Lemma 3.6 The integer $d_{1}$ is the least common multiple of $d_{2}, \ldots, d_{n}$.

Proof Because of equation (2) of lemma 3.5, we may write

$$
\frac{1}{d_{1}}=1-\frac{a}{b}
$$

where $b$ is the least common multiple of $d_{2}, \ldots, d_{n}$. But then $\frac{1}{d_{1}}=\frac{b-a}{b}$ and $b$ divides $d_{1}$ (because $\frac{d_{1}}{d_{i}} \in \mathbb{Z}$ ). Thus $b=d_{1}$ and $b-a=1$.

Lemma 3.7 If $n$ is odd, then all $d_{1}, \ldots, d_{n}$ are squares.

If $n$ is even and $q$ is the square free part of $d_{n}$, then $\frac{d_{1}}{q}, \ldots, \frac{d_{n}}{q}$ are squares in $\mathbb{Z}$.

Proof The determinant of $s$ is an integer and $(\operatorname{det} s)^{2}=d_{1} \cdots d_{n}$. The preceding lemmas tell us that $\frac{d_{1}}{d_{2}}, \ldots, \frac{d_{1}}{d_{n}}$ are integers and squares. So

$$
d_{1}^{n}=d_{1} d_{2} \frac{d_{1}}{d_{2}} \cdots d_{n} \frac{d_{1}}{d_{n}}
$$

is a square and therefore all $d_{i}, 1 \leq i \leq n$ are squares if $n$ is odd. If $n$ is even, this tells us that all square free parts of the $d_{i}$ are equal.

Apart from the abelian groups, there is no character table of a finite group which may be used as Fourier matrix in a modular datum (because of the lack of symmetry). But in analogy to character tables, $d_{1}$ is something like the order of a group and $s_{11}, \ldots, s_{1 n}$ are the degrees of the irreducible characters.

Definition 3.8 We will call $d_{1}, \ldots, d_{n}$ the norms, $s_{11}, \ldots, s_{1 n}$ the degrees and $d_{1}$ the size of the modular datum.

An integral Fourier matrix is not uniquely determined by its degrees, for example: 


\section{Example 2}

$$
\left.\begin{array}{ccccccccccccc}
1 & 1 & 1 & 1 & 2 & 2 & 2 & 4 & 4 & 4 & 8 & 8 & 8 \\
1 & 1 & 1 & 1 & 2 & 2 & 2 & 4 & 4 & 4 & 8 & -8 & -8 \\
1 & 1 & 1 & 1 & 2 & 2 & 2 & 4 & 4 & 4 & -8 & 8 & -8 \\
1 & 1 & 1 & 1 & 2 & 2 & 2 & 4 & 4 & 4 & -8 & -8 & 8 \\
1 & 1 & 1 & 1 & -2 & 2 & 6 & 0 & 0 & -4 & 0 & 0 & 0 \\
1 & 1 & 1 & 1 & 2 & 2 & 2 & -4 & -4 & 4 & 0 & 0 & 0 \\
1 & 1 & 1 & 1 & 6 & 2 & -2 & 0 & 0 & -4 & 0 & 0 & 0 \\
1 & 1 & 1 & 1 & 0 & -2 & 0 & 2 & -2 & 0 & 0 & 0 & 0 \\
1 & 1 & 1 & 1 & 0 & -2 & 0 & -2 & 2 & 0 & 0 & 0 & 0 \\
1 & 1 & 1 & 1 & -2 & 2 & -2 & 0 & 0 & 0 & 0 & 0 & 0 \\
1 & 1 & -1 & -1 & 0 & 0 & 0 & 0 & 0 & 0 & 0 & 0 & 0 \\
1 & -1 & 1 & -1 & 0 & 0 & 0 & 0 & 0 & 0 & 0 & 0 & 0 \\
1 & -1 & -1 & 1 & 0 & 0 & 0 & 0 & 0 & 0 & 0 & 0 & 0
\end{array}\right)
$$

Remark that the first matrix defines negative structure constants. The second one is a congruence multiplication table (see 4) with positive structure constants.

It is a surprising fact that up to dimension 11 there are no other indecomposable integral modular data than example 1 and the Fourier matrix $s$ of the quantum double of $S_{3}$ (listed in the appendix). To be precise, there are 3 permutations of rows of $s$ which give non isomorphic modular data, because they can not be obtained by a simultaneous permutation of rows and columns. In dimension 12 there is (up to independent permutation of rows and columns) exactly one integral Fourier matrix having a $T$-matrix; but it has negative structure constants and the $T$-matrix has infinite order.

\subsection{Integral modular data with a unique norm}

Remember that $d_{1}$ is divisible by all $d_{i}, 1 \leq i \leq n$. 
Lemma 3.9 We have

$$
\sum_{i} s_{i j}^{3} \delta_{i} \equiv \sum_{i} s_{i j}^{2} \delta_{i} \equiv \sum_{i} s_{i j} \delta_{i} \equiv 0\left(\bmod d_{1}\right)
$$

where $\delta_{i}:=d_{1} / d_{i} \in \mathbb{Z}$.

Proof Apply 3.1, (1) to 3.1, (4).

We would like to prove that an integral Fourier matrix $s$ with a unique norm has only \pm 1 as entries. This seems to be true in general:

Conjecture 3.10 If $d_{1}=\ldots=d_{n}$, then $s \in\{ \pm 1\}^{n \times n}$.

Assuming the bound (1) of remark 3.2, this is easy to prove: (remember that the bound holds when the structure constants are nonnegative).

Lemma 3.11 Assume that $\left|s_{i j}\right| \leq s_{1 j}$ for all $i, j$. If $d_{1}=\ldots=d_{n}$, then $s \in\{ \pm 1\}^{n \times n}$.

Proof If $d_{1}=\ldots=d_{n}$, then the matrix $s$ is symmetric and $d_{i}=n$ for all $i$. Then $s_{1 i}=1$ for all $i$, so by our assumption, $\left|s_{i j}\right| \leq 1$ for all $i, j$. But $d_{i}=\sum_{j=1}^{n} s_{i j}^{2}=n$, hence $s_{i j}= \pm 1$ for all $i, j$.

Proposition 3.12 The only dimensions $n$ for which there exist integral modular data $(s, T)$ with $d_{1}=\ldots=d_{n}$ and $\left|s_{i j}\right| \leq s_{1 j}$ for all $i, j$ are powers of 2 .

The corresponding fusion algebras are isomorphic to the group ring of

$$
(\mathbb{Z} / 2 \mathbb{Z})^{k}, \quad n=2^{k} .
$$

Proof By lemma 3.11, integral modular data with $d_{1}=\ldots=d_{n}=n$ and $\left|s_{i j}\right| \leq s_{1 j}$ for all $i, j$ have an $s$-matrix $s$ with entries in $\{ \pm 1\}$. The rows are orthogonal and the first row is $s_{1}:=(1, \ldots, 1)$. Because of all our assumptions, the axiom for integrality of the structure constants gives

$$
\frac{1}{n} \sum_{k} s_{k i} s_{k j} s_{k l} \in \mathbb{Z}
$$

for all $1 \leq i, j, l \leq n$. If we denote the $i$-th column of $s$ by $s_{i}$ and componentwise multiplication of $s_{i}$ and $s_{j}$ by $s_{i} s_{j}$, then this equation says

$$
s_{i} s_{j} \text { is orthogonal to } s_{l}, \quad \text { or } \quad s_{i} s_{j}=s_{l}
$$

(note that $s_{i} s_{j}=-s_{l}$ is impossible because $s_{1 i}=s_{1 j}=s_{1 l}=1$ ). For given $i, j$ there has to be an $l$ such that $s_{i} s_{j}=s_{l}$ because otherwise $s_{i} s_{j}$ would be orthogonal to all elements of an orthogonal basis.

The first row is the unit element with respect to componentwise multiplication, so the set of rows forms an abelian group and all elements of this group have order 2 . 
Remark that there exist symmetric matrices with entries \pm 1 and orthogonal rows for example in dimension 12 and it is conjectured that they exist in all dimension $n=4 k, k \in \mathbb{N}$ (they are called symmetric Hadamard matrices). But in general, the structure constants of the corresponding fusion algebras are rational numbers.

This does not exclude the existence of non isomorphic integral modular data with $d_{1}=\ldots=d_{n}$ in the same dimension; for example:

$$
s_{1}=\left(\begin{array}{cccc}
1 & 1 & 1 & 1 \\
1 & 1 & -1 & -1 \\
1 & -1 & 1 & -1 \\
1 & -1 & -1 & 1
\end{array}\right), \quad s_{2}=\left(\begin{array}{cccc}
1 & 1 & 1 & 1 \\
1 & 1 & -1 & -1 \\
1 & -1 & -1 & 1 \\
1 & -1 & 1 & -1
\end{array}\right) .
$$

Each of $s_{1}, s_{2}$ have 12 different solutions for the matrix $T$.

These matrices give also an example for non uniqueness of a complete decomposition in tensor products:

Let $s$ be the matrix of example 1 (the character table of $\mathbb{Z} / 2 \mathbb{Z}$ ), so $s_{2} \cong s \otimes s$. Then

$$
s \otimes s_{1} \cong s \otimes s_{2}
$$

although $s_{1} \nsucceq s_{2}$ and $s_{1}$ is not a tensor product of smaller symmetric matrices of dimension greater than 1 .

\subsection{Zeros in Fourier matrices}

Lemma 3.13 If an integral modular datum $(s, T)$ has two different norms, then there is a zero in the matrix $s$.

Proof If there are two different norms, then there is an entry of $s$ unequal to \pm 1 , say $s_{k l}$. Suppose that all entries are non zero. Then all norms are greater or equal to $n$ and because $s_{k l} \neq \pm 1, d_{k}>n$. Then

$$
\frac{1}{d_{1}}+\cdots+\frac{1}{d_{n}}<1,
$$

in contradiction to equation (2) of lemma 3.5.

Looking at the matrices of the appendix, one might be led to believe that an integral Fourier matrix has a $T$-matrix only if there is no zero on the diagonal. This is wrong: for example the $S$-matrix of the quantum double of the wreath product $Z_{2}$ 2 $S_{4}$ (the group of monomial $4 \times 4$-matrices with entries \pm 1 ) is integral and has a zero on the diagonal. It is a $325 \times 325$-matrix.

\section{Congruence multiplication tables}

\subsection{Congruences modulo $\sqrt{d_{1}}$}

The computation of all integral Fourier matrices up to dimension 12 provides some interesting observations. One of the most remarkable is that most of them satisfy a certain congruence: 
Definition 4.1 Let $s$ be an integral Fourier matrix, i.e. $s \in \mathbb{Z}^{n \times n}$ and

(1) $s_{i 1}=1$ for $1 \leq i \leq n, \operatorname{det}(s) \neq 0$,

(2) $s s^{T}=\operatorname{diag}\left(d_{1}, \ldots, d_{n}\right)$,

(3) $\sqrt{d_{j}} s_{i j}=\sqrt{d_{i}} s_{j i}$ for $1 \leq i, j \leq n$,

(4) $N_{i j}^{l}:=\sum_{k} \frac{s_{k i} s_{k j} s_{k l}}{d_{k}} \in \mathbb{Z}$ for $1 \leq i, j, l \leq n$.

If in addition $s$ satisfies

(5) $d_{1}$ is a square, $w:=\sqrt{d_{1}} \in \mathbb{N}$,

(6) $g_{i} s_{i j} \equiv g_{i} g_{j}(\bmod w)$ for all $1 \leq i, j \leq n$,

where $g_{i}:=s_{1 i}$ are the degrees of $s$, then we say that $s$ is a congruence multiplication table. Sometimes, we will also need the bound (see 3.2)

(7) $\left|s_{i j}\right| \leq g_{i}$ for all $1 \leq i, j \leq n$.

Define $\mathbb{S}_{i j}:=s_{i j} s_{1 i}$ for $1 \leq i, j \leq n$, so $\mathbb{S}=w S$. Then the congruence (6) takes the following form:

$$
\mathbb{S}_{i j} \equiv g_{i} g_{j}(\bmod w) .
$$

Because $d_{1}$ is the largest norm, the entries of $\mathbb{S}$ are bounded by $w$,

$$
-w<\mathbb{S}_{i j}<w
$$

So in a congruence multiplication table with given degrees $g_{1}, \ldots, g_{n}$, there are at most two possible values for each entry. For a congruence multiplication table $s$, we will always denote the above symmetric matrix by $\mathbb{S}$.

Example 3 Since the degrees divide $w$, one could ask if $g_{i} g_{j}$ is always divisible by $\mathbb{S}_{i j}$. This is true only up to dimension 12 :

$$
s=\left(\begin{array}{ccccccccccccc}
1 & 1 & 1 & 1 & 2 & 8 & 8 & 8 & 10 & 10 & 20 & 20 & 20 \\
1 & 1 & 1 & 1 & 2 & 8 & 8 & 8 & 10 & 10 & 20 & -20 & -20 \\
1 & 1 & 1 & 1 & 2 & 8 & 8 & 8 & 10 & 10 & -20 & 20 & -20 \\
1 & 1 & 1 & 1 & 2 & 8 & 8 & 8 & 10 & 10 & -20 & -20 & 20 \\
1 & 1 & 1 & 1 & 2 & 8 & 8 & 8 & -10 & -10 & 0 & 0 & 0 \\
1 & 1 & 1 & 1 & 2 & 3 & -2 & -2 & 0 & 0 & 0 & 0 & 0 \\
1 & 1 & 1 & 1 & 2 & -2 & 3 & -2 & 0 & 0 & 0 & 0 & 0 \\
1 & 1 & 1 & 1 & 2 & -2 & -2 & 3 & 0 & 0 & 0 & 0 & 0 \\
1 & 1 & 1 & 1 & -2 & 0 & 0 & 0 & -2 & 2 & 0 & 0 & 0 \\
1 & 1 & 1 & 1 & -2 & 0 & 0 & 0 & 2 & -2 & 0 & 0 & 0 \\
1 & 1 & -1 & -1 & 0 & 0 & 0 & 0 & 0 & 0 & 0 & 0 & 0 \\
1 & -1 & 1 & -1 & 0 & 0 & 0 & 0 & 0 & 0 & 0 & 0 & 0 \\
1 & -1 & -1 & 1 & 0 & 0 & 0 & 0 & 0 & 0 & 0 & 0 & 0
\end{array}\right)
$$

is a congruence multiplication table.

Remember that (7) follows if the structure constants are nonnegative. Up to dimension 12, there are 28 integral Fourier matrices with nonnegative structure constants 
and only two of them are not congruence multiplication tables, the character tables of the groups $\mathbb{Z} / 2 \mathbb{Z}$ and $(\mathbb{Z} / 2 \mathbb{Z})^{3}$. For these two matrices, the normalized $S$-matrix is not rational, because the norms are not squares.

In particular, the Fourier matrix of the quantum double of $S_{3}$ is a congruence multiplication table. It seems as if dimension 12 is too small to find more complicated examples, because experiments show that bigger indecomposable Fourier matrices coming from quantum doubles do not satisfy this congruence property anymore.

Also, no character table of a group $(\mathbb{Z} / 2 \mathbb{Z})^{k}, k>3$ is a congruence multiplication table. In this case we have $w=2^{\frac{k}{2}}>2$ and the entries of the matrix $\mathbb{S}$ are \pm 1 . But then $-1 \not \equiv 1(\bmod w)$, so $(6)$ is not satisfied.

However, we may also define $w$ to be the largest integer such that (6) is satisfied. For example the Fourier matrix of the quantum double of $S_{4}$ has the congruence property for $w=\frac{\sqrt{d_{1}}}{2}$.

But let us first consider the case $w=\sqrt{d_{1}}$.

Proposition 4.2 Let $\mathbb{S}$ be a congruence multiplication table and assume also the bound (7). Then

$$
\begin{gathered}
g_{i} g_{j}<\frac{w}{2} \Rightarrow \mathbb{S}_{i j}=g_{i} g_{j}, \\
w \mid g_{i} g_{j} \Rightarrow \mathbb{S}_{i j}=0, \\
g_{i} g_{j}<w \Rightarrow \mathbb{S}_{i j} \geq-\frac{w}{2},
\end{gathered}
$$

for all $1 \leq i, j \leq n$.

Proof Assume that $g_{i} g_{j}<\frac{w}{2}$. Then the two integers congruent to $g_{i} g_{j}$ modulo $w$ in the interval $(-w, w)$ are $g_{i} g_{j}$ and $g_{i} g_{j}-w$. The case $\mathbb{S}_{i j}=g_{i} g_{j}-w$ would imply

$$
g_{i} g_{j}=w-\left|\mathbb{S}_{i j}\right| \geq w-g_{i} g_{j}
$$

because $\mathbb{S}_{i j}<0$ and so $2 g_{i} g_{j} \geq w$ which we excluded at the beginning. The second and third implications are obvious.

The first two implications of the last proposition say that a big part of the matrix is given by the degrees. An interesting problem is to characterize the degrees appearing in congruence multiplication tables. For example, experiments show that there are always at least 2 degrees equal to 1 . We have no proof for this, but we know:

Proposition 4.3 Let $s$ be a congruence multiplication table. Then the number $m$ of degrees of $s$ equal to 1 is less or equal to 4.

If $m>1$, then there is at least one degree equal to $\frac{w}{2}=\sqrt{\frac{d_{1}}{4}}$ (or norm equal to 4 ).

If in addition the structure constants are nonnegative, then $m \in\{1,2,4\}$.

Proof We may assume without loss of generality that $g_{1} \leq \ldots \leq g_{n}$. If there are two degrees equal to 1 then $g_{1}=g_{2}=1$ and the first two rows of $s$ will have norm $d_{1}$. The 
largest possible degree is $\frac{w}{2}$, because it should divide $w$ and the sum of the squares of the degrees is $w^{2}=d_{1}$. Let $r \in \mathbb{Z}$ be maximal such that $g_{n-r+1}, \ldots, g_{n}=\frac{w}{2}$, so $r$ is the number of degrees equal to $\frac{w}{2}$. By proposition 4.2, the first $n-r$ entries of $s$ in the second row are determined by the degrees:

$$
s_{2 i}=g_{i} \quad \text { for } i \leq n-r .
$$

Now the second row has to be orthogonal to the first one, so $r>0$ and $d_{n}=4$. But the sum of the inverse of the norms must be one, so $r \leq 4$ and if $n \neq 4$ then $r<4$.

Since by (6) the values at the end of the second row are congruent $\frac{w}{2}$ modulo $w$, the number of possibilities for the second row to be orthogonal to the first is at most 3 (if $r=3$ ). We get, that the number $m$ of degrees of $s$ equal to 1 is at most 4 .

If the structure constants are nonnegative, then the set of columns with degree 1 forms a group with respect to componentwise multiplication, so the case $m=3$ is excluded.

Proposition 4.4 Let $s$ be a congruence multiplication table of dimension $n$ and with degrees $g_{1}, \ldots, g_{n}$. Then

$$
\sum_{\substack{j=1 \\ 2 g_{i} g_{j}<w}}^{n} g_{j}^{2} \leq \sum_{\substack{j=1 \\ 2 g_{i} g_{j} \geq w}}^{n} g_{j}^{2}
$$

for all $1 \leq i \leq n$.

Proof Use the first implication of proposition 4.2 together with the orthogonality between the first and the $i$-th row.

Proposition 4.5 If $s$ is an integral Fourier matrix of dimension $n$ and of size $d_{1}$ which is a square, then

$$
\tilde{s}:=\left(\begin{array}{cccccccccc}
1 & 1 & 1 & 1 & 2 s_{12} & \cdots & 2 s_{1 n} & b & b & b \\
1 & 1 & 1 & 1 & 2 s_{12} & \cdots & 2 s_{1 n} & b & -b & -b \\
1 & 1 & 1 & 1 & 2 s_{12} & \cdots & 2 s_{1 n} & -b & b & -b \\
1 & 1 & 1 & 1 & 2 s_{12} & \cdots & 2 s_{1 n} & -b & -b & b \\
1 & 1 & 1 & 1 & 2 s_{22} & \cdots & 2 s_{2 n} & 0 & 0 & 0 \\
\vdots & & & \vdots & \vdots & & \vdots & \vdots & & \vdots \\
1 & 1 & 1 & 1 & 2 s_{n 2} & \cdots & 2 s_{n n} & 0 & 0 & 0 \\
1 & 1 & -1 & -1 & 0 & \cdots & 0 & 0 & 0 & 0 \\
1 & -1 & 1 & -1 & 0 & \cdots & 0 & 0 & 0 & 0 \\
1 & -1 & -1 & 1 & 0 & \cdots & 0 & 0 & 0 & 0
\end{array}\right)
$$

where $b:=2 \sqrt{d_{1}}$ is an integral Fourier matrix of dimension $n+6$.

If $s$ is a congruence multiplication table, then $\tilde{s}$ is one as well.

Proof Orthogonality of the rows of $\tilde{s}$ is an easy verification. The size of $\tilde{s}$ is

$$
\tilde{d}_{1}=4+4\left(d_{1}-1\right)+12 d_{1}=16 d_{1}
$$


so the symmetry is obvious as well. For the integrality of the structure constants, we consider all componentwise products of columns; they evidently always yield linear combinations of the columns with integer coefficients. The second assertion is trivial.

Remark 4.6 The contruction of proposition 4.5 is something like an extension of an integral Fourier matrix by $s_{2} \otimes s_{2}$ where $s_{2}$ is the character table of the cyclic group $\mathbb{Z} / 2 \mathbb{Z}$. It is possible to generalize this to an extension by $s_{e} \otimes s_{e}$ where $s_{e}$ is the character table of the cyclic group $\mathbb{Z} / e \mathbb{Z}, e \in \mathbb{N}$. The resulting matrix has $2\left(e^{2}-1\right)$ more columns and is of course not rational anymore (if $e>2$ ). But the structure constants remain rational integers.

For a given matrix $s$, we will denote the matrix $\tilde{s}$ of proposition 4.5 obtained from $s$ by $s^{+6}$.

Example 4 The matrix

$$
s:=\left(\begin{array}{ccccccccccc}
1 & 1 & 1 & 3 & 3 & 3 & 3 & 5 & 5 & 6 & 10 \\
1 & 1 & 1 & 3 & 3 & 3 & 3 & 5 & -10 & 6 & -5 \\
1 & 1 & 1 & 3 & 3 & 3 & 3 & -10 & 5 & 6 & -5 \\
1 & 1 & 1 & -2 & 3 & -2 & -2 & 0 & 0 & 1 & 0 \\
1 & 1 & 1 & 3 & -2 & -2 & -2 & 0 & 0 & 1 & 0 \\
1 & 1 & 1 & -2 & -2 & -2 & 3 & 0 & 0 & 1 & 0 \\
1 & 1 & 1 & -2 & -2 & 3 & -2 & 0 & 0 & 1 & 0 \\
1 & 1 & -2 & 0 & 0 & 0 & 0 & -1 & -1 & 0 & 1 \\
1 & -2 & 1 & 0 & 0 & 0 & 0 & -1 & -1 & 0 & 1 \\
1 & 1 & 1 & \frac{1}{2} & \frac{1}{2} & \frac{1}{2} & \frac{1}{2} & 0 & 0 & -\frac{3}{2} & 0 \\
1 & -\frac{1}{2} & -\frac{1}{2} & 0 & 0 & 0 & 0 & \frac{1}{2} & \frac{1}{2} & 0 & -\frac{1}{2}
\end{array}\right)
$$

is not an integral Fourier matrix, because its structure constants are not all integers (and because its entries are not all integers). But the construction of proposition 4.5 applied to $s$ yields a $17 \times 17$-matrix which is a congruence multiplication table.

Theorem 1 There exist congruence multiplication tables in all dimensions except 2 , $3,5,6$.

Proof In the appendix, we give congruence multiplication tables for the dimensions $n=4,7,8,9,10,11$ and 12 . By proposition 4.5, they exist in all dimensions greater than 12 . To exclude the cases 2,3,5 and 6 we have computed all integral Fourier matrices in these dimensions.

4.2 The modulus of an integral Fourier matrix

Now we consider the case where $w$ is not necessarily equal to $\sqrt{d_{1}}$.

Definition 4.7 Let $s$ be an integral Fourier matrix. Then we call the greatest integer $w \in \mathbb{Z}_{>0}$ such that

$$
g_{i} s_{i j} \equiv g_{i} g_{j}(\bmod w) \quad \text { for all } 1 \leq i, j \leq n,
$$


where $g_{i}:=s_{1 i}$ are the degrees of $s$, the modulus $\mathrm{w}(s):=w$ of $s$. We set $\mathrm{w}((1)):=$ $\infty, a \equiv b(\bmod \infty): \Leftrightarrow a=b$, and $\operatorname{gcd}(a, \infty):=a$ for all $a, b \in \mathbb{Z}$.

Proposition 4.8 If $s$ and $s^{\prime}$ are integral Fourier matrices, then

$$
\mathrm{w}\left(s \otimes s^{\prime}\right)=\operatorname{gcd}\left(\mathrm{w}(s), \mathrm{w}\left(s^{\prime}\right)\right) .
$$

Proof Let $n, n^{\prime}$ be the dimensions of $s, s^{\prime}$. The rows and columns of the tensor product $s \otimes s^{\prime}$ are indexed by pairs $\left(i_{1}, i_{2}\right), 1 \leq i_{1} \leq n, 1 \leq i_{2} \leq n^{\prime}$ and an entry is

$$
\left(s \otimes s^{\prime}\right)_{\left(i_{1}, i_{2}\right),\left(j_{1}, j_{2}\right)}=s_{i_{1} j_{1}} s_{i_{2} j_{2}}^{\prime} .
$$

Then

$$
g_{i_{1}} g_{i_{2}}^{\prime} s_{i_{1} j_{1}} s_{i_{2} j_{2}}^{\prime} \equiv g_{i_{1}} g_{i_{2}}^{\prime} g_{j_{1}} g_{j_{2}}^{\prime}\left(\bmod \mathrm{w}\left(s \otimes s^{\prime}\right)\right)
$$

and this holds in particular for $i_{1}=j_{1}=1$ and $i_{2}=j_{2}=1$ from which follows that $\mathrm{w}\left(s \otimes s^{\prime}\right)$ divides $\tilde{w}:=\operatorname{gcd}\left(\mathrm{w}(s), \mathrm{w}\left(s^{\prime}\right)\right)$. On the other hand,

$$
\begin{aligned}
& g_{i} s_{i j} \equiv g_{i} g_{j}(\bmod \tilde{w}) \quad \text { for all } 1 \leq i, j \leq n, \\
& g_{i}^{\prime} s_{i j}^{\prime} \equiv g_{i}^{\prime} g_{j}^{\prime}(\bmod \tilde{w})
\end{aligned}
$$

so $g_{i_{1}} g_{i_{2}}^{\prime} s_{i_{1} j_{1}} s_{i_{2} j_{2}}^{\prime} \equiv g_{i_{1}} g_{i_{2}}^{\prime} g_{j_{1}} g_{j_{2}}^{\prime}(\bmod \tilde{w})$ and hence $\tilde{w} \leq \mathrm{w}\left(s \otimes s^{\prime}\right)$.

The set $\mathcal{F}$ of integral Fourier matrices up to isomorphisms forms a monoid together with tensor product. The preceding proposition states that

$$
\mathrm{w}:(\mathcal{F}, \otimes) \rightarrow(\mathbb{N} \cup\{\infty\}, \mathrm{gcd}), s \mapsto \mathrm{w}(s)
$$

is a monoid homomorphism.

\section{Integral modular data with two different degrees}

\subsection{Some general observations}

In this section we will try to find out how integral Fourier matrices with two different degrees look like. If the structure constants are positive, then the columns of degree 1 form a group isomorphic to a direct product of cyclic groups $\mathbb{Z} / 2 \mathbb{Z}$, so the number of degrees equal to 1 is a power of 2 , say $2^{m}, m \in \mathbb{Z}_{\geq 0}$. If there are only two different values 1 and $g$ for the degrees, then $d_{1}=2^{m}+q g^{2}$ for some $q \in \mathbb{Z}$. This implies that $g^{2}$ divides $2^{m}$, because $g^{2}$ divides $d_{1}$. So $g=2^{k}$ for some $k \in \mathbb{Z}$ with $m \geq 2 k$.

Let $c$ be the character table of $(\mathbb{Z} / 2 \mathbb{Z})^{m}$. Then the first $2^{m}$ columns of the matrix $s$ build a $\left(2^{m}+q\right) \times 2^{m}$-matrix. Let $a_{1}, \ldots, a_{2^{m}}$ be the multiplicities of the rows of this matrix; so the $i$-th row of $c$ appears $a_{i}$ times. 
Let us first assume that $a_{1}=2^{m}$. This holds for example in the infinite sequence of modular data of section 5.2. Then the symmetry and orthogonality of the Fourier matrix give

$$
a_{1}+\sum_{i=2}^{2^{m}} c_{i j} a_{i} g^{2}=0
$$

for all $1<j \leq 2^{m}$ and

$$
a_{1}+\sum_{i=2}^{2^{m}} a_{i} g^{2}=2^{m}+q 2^{2 k}
$$

or equivalently

$$
\left(2^{m-2 k}, a_{2}, \ldots, a_{2^{m}}\right) c=\left(2^{m-2 k}+q, 0, \ldots, 0\right) .
$$

But $c^{-1}=2^{-m} c$ and the first row of $c$ has only 1's, so

$$
q=\left(2^{m}-1\right) 2^{m-2 k}, \quad a_{2}=\cdots=a_{2^{m}}=2^{m-2 k} .
$$

Now we return to the general case where $a_{1}$ is arbitrary. Let $0 \leq v<2^{m}$ be such that

$$
\sum_{i=1}^{v} a_{i} \leq 2^{m}, \quad-2^{m}+\sum_{i=1}^{v+1} a_{i}=: \delta>0 .
$$

Then

$$
\left(\sum_{i=1}^{\nu} a_{i} c_{i j}\right)+\left(a_{\nu+1}-\delta+\delta g^{2}\right) c_{\nu+1, j}+\sum_{i=\nu+2}^{2^{m}} a_{i} c_{i j} g^{2}=\delta_{i j}\left(2^{m}+q g^{2}\right) .
$$

By the same argument as above, we get

$$
\begin{aligned}
a_{1}, \ldots, a_{v} & =\left(2^{m}+q g^{2}\right) 2^{-m}, \\
a_{v+1} & =\left(2^{m}+q g^{2}\right) 2^{-m}+\delta-\delta g^{2}, \\
a_{v+2}, \ldots, a_{2^{m}} & =\left(2^{m-2 k}+q\right) 2^{-m} .
\end{aligned}
$$

Because $a_{1}, \ldots, a_{v}$ are integers, $2^{m-2 k}$ divides $q$ say $q=q^{\prime} 2^{m-2 k}$. By the third equality, $2^{2 k}$ divides $1+q^{\prime}$, so $2^{2 k} \leq 1+q^{\prime}$ and therefore

$$
v \leq \frac{2^{m}}{a_{v}}=\frac{2^{2 m}}{2^{m}+q g^{2}}=\frac{2^{m}}{1+q^{\prime}} \leq 2^{m-2 k} .
$$

Unfortunately, in a certain sense, there is no better bound: 
Example 5 The matrix

$$
\left(\begin{array}{cccccccccccccc}
1 & 1 & 1 & 1 & 1 & 1 & 1 & 1 & 2 & 2 & 2 & 2 & 2 & 2 \\
1 & 1 & 1 & 1 & 1 & 1 & 1 & 1 & 2 & 2 & -2 & -2 & -2 & -2 \\
1 & 1 & 1 & 1 & 1 & 1 & 1 & 1 & -2 & -2 & 2 & 2 & -2 & -2 \\
1 & 1 & 1 & 1 & 1 & 1 & 1 & 1 & -2 & -2 & -2 & -2 & 2 & 2 \\
1 & 1 & 1 & 1 & -1 & -1 & -1 & -1 & 2 & -2 & 2 & -2 & 2 & -2 \\
1 & 1 & 1 & 1 & -1 & -1 & -1 & -1 & 2 & -2 & -2 & 2 & -2 & 2 \\
1 & 1 & 1 & 1 & -1 & -1 & -1 & -1 & -2 & 2 & 2 & -2 & -2 & 2 \\
1 & 1 & 1 & 1 & -1 & -1 & -1 & -1 & -2 & 2 & -2 & 2 & 2 & -2 \\
1 & 1 & -1 & -1 & 1 & 1 & -1 & -1 & 0 & 0 & 0 & 0 & 0 & 0 \\
1 & 1 & -1 & -1 & -1 & -1 & 1 & 1 & 0 & 0 & 0 & 0 & 0 & 0 \\
1 & -1 & 1 & -1 & 1 & -1 & 1 & -1 & 0 & 0 & 0 & 0 & 0 & 0 \\
1 & -1 & 1 & -1 & -1 & 1 & -1 & 1 & 0 & 0 & 0 & 0 & 0 & 0 \\
1 & -1 & -1 & 1 & 1 & -1 & -1 & 1 & 0 & 0 & 0 & 0 & 0 & 0 \\
1 & -1 & -1 & 1 & -1 & 1 & 1 & -1 & 0 & 0 & 0 & 0 & 0 & 0
\end{array}\right)
$$

is the tensor product of the matrix of dimension 2 and the matrix of dimension 7 of the appendix. We have

$$
m=3, \quad k=1, \quad q=5, \quad v=2, \quad \delta=0, \quad a_{1}=a_{2}=4, \quad a_{3}, \ldots, a_{8}=1 .
$$

Remark also that this is not a congruence multiplication table.

5.2 An infinite sequence of integral modular data with rational $T$-matrix and different degrees

The modular datum given by the quantum double of the dihedral group with 8 elements has the above properties. By permuting two columns in the Fourier matrix we get a Fourier matrix which has a rational $T$-matrix of finite order (this is excluded in $T$-matrices of quantum doubles of non abelian groups, because the order of $T$ is exactly the exponent of the corresponding group). We generalize this and get the following sequence.

Proposition 5.1 Let $k \in \mathbb{Z}$,

$$
m:=2 k+1, \quad g:=2^{k}, \quad q:=2\left(2^{m}-1\right), \quad a:=2^{m},
$$

and

$$
c:=\left(\begin{array}{cc}
1 & 1 \\
1 & -1
\end{array}\right) \otimes \cdots \otimes\left(\begin{array}{cc}
1 & 1 \\
1 & -1
\end{array}\right)
$$


be the $m$-th tensor power of the character table of $\mathbb{Z} / 2 \mathbb{Z}$. Then

$$
S:=\frac{1}{a}\left(\begin{array}{cccccccc}
1 & \cdots & 1 & g c_{21} & g c_{21} & \cdots & g c_{a 1} & g c_{a 1} \\
\vdots & & \vdots & \vdots & \vdots & & \vdots & \vdots \\
1 & \cdots & 1 & g c_{2 a} & g c_{2 a} & \cdots & g c_{a a} & g c_{a a} \\
g c_{21} & \cdots & g c_{2 a} & g^{2} & -g^{2} & & & \\
g c_{21} & \cdots & g c_{2 a} & -g^{2} & g^{2} & & 0 & \\
\vdots & & \vdots & & & \ddots & & \\
g c_{a 1} & \cdots & g c_{a a} & & 0 & & g^{2} & -g^{2} \\
g c_{a 1} & \cdots & g c_{a a} & & & & -g^{2} & g^{2}
\end{array}\right)
$$

and

$$
T=\operatorname{diag}(\underbrace{1, \ldots, 1}_{a}, \underbrace{1,-1, \ldots, 1,-1}_{2(a-1)})
$$

define a modular datum of dimension $2\left(3 \cdot 2^{2 k}-1\right)$ with rational matrices $S$ and $T$.

The structure constants are nonnegative and their greatest value is $2^{k-1}$.

Proof Orthogonality of the rows of $S$ is an easy verification (use several times the fact that $c$ is orthogonal).

We want to prove that the structure constants are nonnegative rational integers. For this, consider the matrix $s$ which is obtained by dividing each row of $S$ by its first entry. Then the fusion algebra is the $\mathbb{Z}$-lattice spanned by the columns $v_{1}, \ldots, v_{a}, w_{1}, \ldots, w_{2 a-2}$ of $s$ and multiplication is componentwise. Products of the form $v_{i} v_{j}$ or $v_{i} w_{j}$ give columns of $s$, so the only interesting cases are $w_{i} w_{j}$. Without loss of generality, we may restrict to the three cases $w_{1} w_{1}, w_{1} w_{2}$ and $w_{1} w_{3}$. One easily sees that

$$
w_{1} \cdot w_{1}=\sum_{\substack{i=1 \\ s_{a+1, i}=1}}^{a} v_{i}, \quad w_{1} \cdot w_{2}=\sum_{\substack{i=1 \\ s_{a+1, i}=-1}}^{a} v_{i},
$$

and that

$$
w_{1} \cdot w_{3}=2^{k-1}\left(w_{j}+w_{j+1}\right) \text { for some } 1 \leq j \leq 2 a-2 .
$$

It remains to verify $(S T)^{3}=1$. A tiring calculation gives $(S T)^{2}=T S$, which suffices because $T^{2}=S^{2}=1$.

Remark 5.2 The subgroup $\langle S, T\rangle \leq \mathrm{GL}_{2\left(3 \cdot 2^{2 k}-1\right)}(\mathbb{Q})$ spanned by the matrices $S$ and $T$ is isomorphic to the symmetric group $S_{3}$.

The proof of the following proposition for arbitrary $k$ has been suggested by the referee.

Proposition 5.3 The fusion algebras of the $S$-matrices of Proposition 5.1 for $k>1$ are not isomorphic to the fusion algebras associated to quantum doubles of finite groups. 
Proof Equation (3) of section 6 gives a definition for the matrices $S$ and $T$. They are indexed by pairs $(\bar{g}, \chi)$, where $\bar{g}$ is a conjugacy class of $G$ and $\chi$ is an irreducible character of the centralizer of $g$ in $G$. In particular, the column of $S$ indexed by $(\bar{e}, 1)$ is the Perron-Frobenius vector, this is the unique column of $S$ with positive real entries. These entries are

$$
S_{(\bar{h}, \chi),(\bar{e}, 1)}=\frac{\operatorname{deg}(\chi)}{\left|C_{G}(h)\right|}
$$

and if our $S$-matrix was associated to the quantum double of a finite group $G$, then these would be the values $\frac{1}{a}, \ldots, \frac{1}{a}, \frac{g}{a}, \ldots, \frac{g}{a}$, where $\frac{1}{a}$ occurs $a$ times and $\frac{g}{a}$ occurs $2(a-1)$ times. It follows immediately that $G$ has $a$ elements and is nonabelian.

Now if $h$ does not lie in the center $Z$ of $G$, then its centralizer will have less than $a$ elements and since $S_{(\bar{h}, 1),(\bar{e}, 1)}=\left|C_{G}(h)\right|^{-1}$, we get $\left|C_{G}(h)\right|=\frac{a}{g}$. This also implies that the centralizer is abelian because all degrees of irreducible characters will be 1. Hence the $a$ values $\frac{1}{a}$ correspond to pairs $(\bar{h}, \chi)$ with $h \in Z$ and $\operatorname{deg}(\chi)=1$. So the number of 1-dimensional irreducible representations of $G$ is $a /|Z|$. Further, all irreducible representations of $G$ have dimension 1 or $g$.

Denote by $n_{g}$ the number of irreducible representations of $G$ of dimension $g$. Then $a=a /|Z|+g^{2} n_{g}$, i.e. $|Z|=2$ and $n_{g}=1$. This implies that the number of conjugacy classes of $G$ is $2^{2 k}+1$ and therefore we have $2^{2 k}-1$ classes which are not in $Z$. Finally we obtain

$$
a=2^{2 k+1}=|G|=|Z|+\left(2^{2 k}-1\right) \frac{a}{2^{k+1}}=2+2^{3 k}-2^{k}
$$

which is impossible for $k>1$.

\section{Some infinite sequences of integral finite group modular data}

For a finite group $G$, the $S$ - and $T$-matrices of the quantum double of $G$ are indexed by pairs $(\bar{g}, \chi)$, where $\bar{g}$ is a conjugacy class of $G$ and $\chi$ is an irreducible character of the centralizer of $g$ in $G$ (choose one representative for each class). Its entries are given by

$$
\begin{gathered}
S_{(\bar{a}, \chi),\left(\bar{b}, \chi^{\prime}\right)}:=\frac{1}{\left|C_{G}(a)\right|\left|C_{G}(b)\right|} \sum_{\substack{g \in G \\
a g b g^{-1}=g b g^{-1} a}} \overline{\chi\left(g b g^{-1}\right) \chi^{\prime}\left(g^{-1} a g\right),} \\
T_{(\bar{a}, \chi),\left(\bar{a}^{\prime}, \chi^{\prime}\right)}:=\delta_{\bar{a}, \bar{a}^{\prime}} \delta_{\chi, \chi^{\prime}} \frac{\chi(a)}{\chi(e)}
\end{gathered}
$$

where $C_{G}(a)$ is the centralizer of $a$ in $G$ and $e$ is the neutral element of $G$.

Most indecomposable integral modular data known to the author have fusion algebras isomorphic to the fusion algebra corresponding to the $S$-matrix of the quantum double of a finite group, so they have an $S$-matrix which is of the above form up to an independent permutation of rows and columns. However, the 2-dimensional modular data of example 1 and the infinite sequence of proposition 5.1 do not have this form. 
For example, there are 6 non isomorphic $S$-matrices with fusion algebras isomorphic to the algebra of the quantum double of $S_{3}$. Four of them have $T$-matrices which make them to modular data, but the groups spanned by $S$ and $T$ fall into two isomorphism classes, the one corresponding to the quantum double is $\operatorname{PSL}_{2}(\mathbb{Z} / 6 \mathbb{Z})$ and has 72 elements, the other one is $\mathrm{SL}_{2}(\mathbb{Z} / 12 \mathbb{Z}) / N$ where $N$ is a normal subgroup of order 4 (it has 288 elements).

The following proposition has been pointed out to the author by the referee and by Gannon after the present paper had been submitted:

Proposition 6.1 Let $G$ be a finite group and $S$ be the $S$-matrix of the quantum double of $G$. If $S$ is rational, then $G$ is solvable and the only possible composition factors are the cyclic groups $Z_{2}$ and $Z_{3}$.

Proof This is a consequence of the Galois symmetry of modular data mentioned in [2] or [6]: restricted to the finite group modular data, it says that for any Galois automorphism $\sigma_{\ell}$ in $\operatorname{Gal}\left(Q\left[\zeta_{m}\right] / Q\right)=Z_{m}^{\times}$, where $\mathbb{Q}\left[\zeta_{m}\right]$ is the $m$-th cyclotomic field and $m$ is the exponent of $G$, there is a permutation (also denoted by $\sigma_{\ell}$ ) of the indices $1, \ldots, n$ such that

$$
\sigma_{\ell}\left(S_{i j}\right)=S_{\sigma_{\ell}(i), j}, \quad T_{\sigma_{\ell}(i), \sigma_{\ell}(i)}=T_{i i}^{\ell^{2}}
$$

The rationality of $S$ implies that the permutation is trivial on the indices, so $T_{i i}^{\ell^{2}}=T_{i i}$ for all $\ell$ coprime to $m$ ( $T_{i i}$ will be $m$-th roots of unity). This requires that $m$ divides 24 . Now by Burnside's $p^{a} q^{b}$ theorem, such a $G$ must be solvable, so the only possible composition factors are $Z_{2}$ and $Z_{3}$.

In the next section, we give some infinite sequences of integral modular data arising as subdirect products. The following list contains the groups with at most 100 elements and rational $S$-matrix which are not included in our sequences of subdirect products and are not direct products of smaller groups. A pair $(a, b)$ corresponds to the $b$-th group of order $a$ in the database of small groups of the computer algebra system GAP:

$(32,49),(32,50),(64,134),(64,137),(64,138),(64,177),(64,178),(64,182)$.

\subsection{Subdirect products}

We consider subdirect products with certain properties. Let $H$ be a finite group, $N$ a normal subgroup and $\mu: H \rightarrow H / N$ the canonical projection. The subgroup $G$ of the direct product $H^{r}$ consisting of all $\left(g_{1}, \ldots, g_{r}\right)$ such that $\mu\left(g_{i}\right)=\mu\left(g_{j}\right)$ for all $1 \leq i, j \leq r$ is the subdirect product $G=H \times_{\mu} \cdots \times_{\mu} H$.

Example 6 Take $H=S_{3}$ and $N=Z_{3}$. Then the group $G$ has $\frac{1}{2}\left(3^{r}+3\right)$ conjugacy classes. The centralizer of an element of order 2 in $G$ is $Z_{2}$ and the centralizer of a non trivial element in $Z_{3}^{r}$ is $Z_{3}^{r}$, so the $S$-matrix of the quantum double of $G$ has dimension $\frac{1}{2}\left(3^{2 r}+7\right)$. 
We will prove that the $S$-matrix of the quantum double of subdirect products of the above form is rational in the following cases:

(1) $H=S_{3}, D_{4}, Q_{8}$ and arbitrary normal subgroup $N$,

(2) $H=S_{4}$ and $N \neq A_{4}$.

To prove this for the case $H=S_{4}$, we need a technical proposition and a computer program (which is still simpler than determining all conjugacy classes and centralizers of the subdirect products in details). The other cases may also be proved using the same technique, but there are also easier arguments which we do not want to keep back.

The principle is always the same: To each summand in the formula (3) for the $S$ matrix, we find another summand which is its complex conjugate. This suffices for the groups whose exponent divides 6 . For $S_{4}$, we have to consider more automorphisms.

6.2 The case $H=D_{4}$

For the dihedral group, we use the following lemma:

Lemma 6.2 Let $N \unlhd H$ be finite groups and $G=H \times_{\mu} \cdots \times_{\mu} H$ be the subdirect product with respect to $\mu: H \rightarrow H / N$. Assume that there exists a $\sigma \in H$ such that

$$
\tau^{\sigma}:=\sigma^{-1} \tau \sigma=\tau^{-1}
$$

for all $\tau \in N$. Then for the $S$-matrix of the quantum double of $G$ we have

$$
S_{\left(\overline{\left(a_{1}, \ldots, a_{r}\right)}, \chi\right),\left(\overline{\left(a_{1}^{\prime}, \ldots, a_{r}^{\prime}\right)}, \chi^{\prime}\right)} \in \mathbb{R}
$$

for $\left(a_{1}, \ldots, a_{r}\right),\left(a_{1}^{\prime}, \ldots, a_{r}^{\prime}\right) \in G$ with $a_{i}, a_{i}^{\prime} \in N$ for all $1 \leq i \leq r$ and any $\chi \in$ $\operatorname{Irr}\left(C_{G}\left(\left(a_{1}, \ldots, a_{r}\right)\right)\right), \chi^{\prime} \in \operatorname{Irr}\left(C_{G}\left(\left(a_{1}^{\prime}, \ldots, a_{r}^{\prime}\right)\right)\right)$.

Further, the entry $S_{\left(\overline{\left(a_{1}, \ldots, a_{r}\right)}, \chi\right),\left(\overline{\left(a_{1}^{\prime}, \ldots, a_{r}^{\prime}\right)}, \chi^{\prime}\right)}$ is rational if $a_{1}, \ldots, a_{r}$ lie in $N$, $a_{1}^{\prime}, \ldots, a_{r}^{\prime}$ are arbitrary and $\chi^{\prime}$ is a rational character.

Proof Let $a_{i}, b_{i} \in N$ and $g_{i} \in H$ be such that $a_{i} g_{i} b_{i} g_{i}^{-1}=g_{i} b_{i} g_{i}^{-1} a_{i}$ for $1 \leq i \leq r$. Then for $h_{i}:=\sigma^{-1} g_{i}$ we get

$$
h_{i} b_{i} h_{i}^{-1}=g_{i} b_{i}^{-1} g_{i}^{-1}, \quad h_{i}^{-1} a_{i} h_{i}=g_{i}^{-1} a_{i}^{-1} g_{i}, \quad a_{i} h_{i} b_{i} h_{i}^{-1}=h_{i} b_{i} h_{i}^{-1} a_{i},
$$

and if $\left(g_{1}, \ldots, g_{r}\right) \in G$ then $\left(h_{1}, \ldots, h_{r}\right) \in G$. So in the formula for $S$, we can pair the summands

$$
\chi\left(\underline{g}_{\underline{b}} \underline{g}^{-1}\right) \chi^{\prime}\left(\underline{g}^{-1} \underline{a} \underline{g}\right)+\chi\left(\underline{h b h}^{-1}\right) \chi^{\prime}\left(\underline{h}^{-1} \underline{a h}\right) \in \mathbb{R}
$$

where $g=\left(g_{1}, \ldots, g_{r}\right)$ etc.

For the second assertion, use exactly the same argument.

Remark 6.3 If $G$ is a finite group, $|G|=k$ and $\sigma \in G$, then $\chi(\sigma)$ is rational for every character $\chi$ of $G$ if and only if $\sigma$ is conjugate to $\sigma^{m}$ for every integer $m$ prime to $k$. 
Example 7 Let $G=H \times_{\mu} \cdots \times_{\mu} H$ be the subdirect product with $r$ factors where $H=D_{4}$ is the dihedral group with 8 elements and $\mu$ is the projection $H \rightarrow H / N$ where $N=Z_{4}$ is the cyclic normal subgroup of $D_{4}$ of order 4 . Let $\eta$ be a generator of $N$.

For all $\sigma \in H \backslash N$ and $\tau \in N$ we have $\sigma^{2}=1$ and $\tau^{\sigma}=\tau^{-1}$ (therefore also $\sigma^{\tau}=$ $\left.\tau^{2} \sigma\right)$. Further, $\sigma^{\sigma^{\prime}} \in\left\{\sigma, \eta^{2} \sigma\right\}$ for all $\sigma, \sigma^{\prime} \in H \backslash N$ and $N \times \ldots \times N \leq G$ is abelian and normal in $G$. So we get $2^{r}+\frac{1}{2}\left(4^{r}-2^{r}\right)$ conjugacy classes of $G$ of the form

$$
\left\{\left(\eta_{1}, \ldots, \eta_{r}\right),\left(\eta_{1}, \ldots, \eta_{r}\right)^{-1}\right\}
$$

with $\eta_{1}, \ldots, \eta_{r} \in N$, and $2^{r}$ conjugacy classes of the form

$$
\left\{\left(\eta_{1}^{2} \sigma_{1}, \ldots, \eta_{r}^{2} \sigma_{r}\right) \mid \eta_{1}, \ldots, \eta_{r} \in N\right\}
$$

with $\sigma_{1}, \ldots, \sigma_{r} \in H \backslash N$. Hence $G$ has $2^{r+1}+2^{2 r-1}-2^{r-1}$ conjugacy classes.

The centralizer of $\left(\eta_{1}, \ldots, \eta_{r}\right) \in N \times \ldots \times N$ are:

$$
C_{G}\left(\left(\eta_{1}, \ldots, \eta_{r}\right)\right)= \begin{cases}G & \text { if } \eta_{i} \in\left\{1, \eta^{2}\right\} \\ N \times \ldots \times N & \text { else. }\end{cases}
$$

The centralizer of $\left(\sigma_{1}, \ldots, \sigma_{r}\right) \in H \backslash N \times \ldots \times H \backslash N$ are:

$$
\begin{aligned}
& C_{G}\left(\left(\sigma_{1}, \ldots, \sigma_{r}\right)\right) \\
& \quad=\left\{\left(\tau_{1}, \ldots, \tau_{r}\right) \mid\left(\tau_{i} \in\left\{1, \eta^{2}\right\} \quad \forall i\right) \text { or }\left(\tau_{i} \in H \backslash N \text { and } \sigma_{i}^{\tau_{i}}=\sigma_{i} \quad \forall i\right)\right\} \\
& \quad \cong Z_{2}^{r+1}
\end{aligned}
$$

because $C_{G}\left(\left(\sigma_{1}, \ldots, \sigma_{r}\right)\right)$ is a subgroup of order $2^{r+1}$ of

$$
C_{H}\left(\sigma_{1}\right) \times \ldots \times C_{H}\left(\sigma_{r}\right) \cong\left(Z_{2} \times Z_{2}\right)^{r}
$$

which is a group of exponent 2 .

The rationality of the $S$-matrix of the quantum double now follows from lemma 6.2:

Proposition 6.4 The finite group modular data associated to $G$ is integral for all $r \in \mathbb{N}$.

Proof We only need to check that all characters of $G$ are rational, because then we know that non rational characters of centralizers only appear in the case of conjugacy classes lying in $N \times \ldots \times N$. And these are handled by lemma 6.2.

But by remark 6.3, it suffices to prove that an element of $G$ is conjugate to all its odd powers $\left(|G|=2^{2 r+1}\right)$. This follows from $\sigma^{3}=\sigma$ and $\tau^{\sigma}=\tau^{-1}$ for $\sigma \in H \backslash N$, $\tau \in N$. 
6.3 The case $H=Q_{8}, N \neq H$

For a finite group $G$, define

$$
k_{G}(a, b):=\left|\left\{g \in G \mid a g b g^{-1}=g b g^{-1} a\right\}\right|
$$

to be the number of summands in the sum of formula (3) for the $S$-matrix of the quantum double.

The conjugacy classes are similar to those in Example 7. The number of elements of the centralizer also coincide. There are two important differences: The elements of $H \backslash N$ do not necessarily have order 2 and their centralizer are not necessarily isomorphic to $Z_{2}$. But the following holds:

Proposition 6.5 Let $H:=Q_{8}$ and $N \unlhd H, N \neq H$ be a normal subgroup.

Then $k_{H}(a, b) \in\{0,|H|\}$ for all $a, b \in H$ and we have bijections

$$
\varphi_{\sigma}: H \rightarrow H
$$

for $\sigma \in H$ such that $\varphi_{\sigma}(h) \neq h$ for all $h \in H$ and:

$$
\begin{gathered}
\forall b, g \in H, a \in N \sigma \text { with } k_{H}(a, b) \neq 0: \\
g b g^{-1}=\varphi_{\sigma}(g) b^{-1} \varphi_{\sigma}(g)^{-1} \text { and } g^{-1} a g=\varphi_{\sigma}(g)^{-1} a^{-1} \varphi_{\sigma}(g) .
\end{gathered}
$$

Proof Compute $k_{H}(a, b)$ for all $a, b \in H$ and $\varphi_{\sigma}$ explicitly.

Now let $G$ be the subdirect product $H \times_{\mu} \ldots \times_{\mu} H$ with $r$ factors where $\mu: H \rightarrow$ $H / N$ is the canonical projection, $H=Q_{8}$ and $N$ is a normal subgroup of $H, N \neq H$. Then by proposition 6.5 , for all $\underline{a}, \underline{b}, g \in G, \chi \in \operatorname{Irr}\left(C_{G}(\underline{a})\right)$ and $\chi^{\prime} \in \operatorname{Irr}\left(C_{G}(\underline{b})\right)$,

$$
\overline{\chi\left(\underline{g} \underline{b}^{-1}\right) \chi^{\prime}\left(\underline{g}^{-1} \underline{a} \underline{g}\right)}=\chi\left(\underline{\varphi_{\sigma}}(\underline{g}) \underline{b} \underline{\varphi_{\sigma}}(\underline{g})^{-1}\right) \chi^{\prime}\left(\underline{\varphi_{\sigma}}(\underline{g})^{-1} \underline{a} \underline{\varphi_{\sigma}}(\underline{g})\right)
$$

where $\sigma \in H$ is such that $\underline{a} \in N \sigma \times \ldots \times N \sigma$ and $\varphi_{\sigma}:=\left(\varphi_{\sigma}, \ldots, \varphi_{\sigma}\right)$. So in the sum of formula (3), we can always pair $g$ with $\underline{\varphi_{\sigma}}(g)$ and obtain a rational number. We have proved:

Proposition 6.6 The finite group modular data associated to $G$ is integral for all $r \in \mathbb{N}$.

6.4 The cases $H=S_{3}, N$ arbitrary and $H=S_{4}, N=Z_{2} \times Z_{2}$

For $H=S_{3}$, the situation is simple; we have almost done the work in example 6 and therefore leave out a direct proof here.

More generally, let $H$ be a finite group and $N \unlhd H$ be a normal subgroup. For $a, b \in H$, define

$$
K_{H}(a, b):=\left\{g \in H \mid a g b g^{-1}=g b g^{-1} a\right\}
$$

to be the set of $g$ appearing in the sum of formula (3) for the $S$-matrix of the quantum double of $H$. 
Proposition 6.7 Assume that for all $m$ coprime to the exponent of $H$, there exists for all $a, b \in H$ a bijective

$$
\varphi_{a, b}^{(m)}: K_{H}(a, b) \rightarrow K_{H}(a, b)
$$

such that $\varphi_{a, b}^{(m)}(g) \neq g$ and

$$
g b g^{-1}=\varphi_{a, b}^{(m)}(g) b^{m} \varphi_{a, b}^{(m)}(g)^{-1}, \quad g^{-1} a g=\varphi_{a, b}^{(m)}(g)^{-1} a^{m} \varphi_{a, b}^{(m)}(g)
$$

for all $a, b \in H, g \in K_{H}(a, b)$.

Further, assume that for all $a^{\prime}, b^{\prime} \in H, g^{\prime} \in K_{H}\left(a^{\prime}, b^{\prime}\right)$, the equalities

$$
N a=N a^{\prime}, \quad N b=N b^{\prime}, \quad N g=N g^{\prime}
$$

imply $N \varphi_{a, b}^{(m)}(g)=N \varphi_{a^{\prime}, b^{\prime}}^{(m)}\left(g^{\prime}\right)$.

Then the $S$-matrix of the quantum double of the subdirect product $G:=H \times \mu$ $\ldots \times{ }_{\mu} H$, where $\mu: H \rightarrow H / N$ is the canonical projection, is rational.

Proof Let $\underline{a}=\left(a_{1}, \ldots, a_{r}\right), \underline{b}=\left(b_{1}, \ldots, b_{r}\right), \underline{g}=\left(g_{1}, \ldots, g_{r}\right) \in G$ with $g_{i} \in$ $K_{H}\left(a_{i}, b_{i}\right)$, and denote the set of such $g$ by $\underline{K}_{H}(\underline{a}, \underline{b})$. By our assumptions,

$$
\underline{\varphi_{a, b}^{(m)}}:=\left(\varphi_{a_{1}, b_{1}}^{(m)}, \ldots, \varphi_{a_{r}, b_{r}}^{(m)}\right)
$$

is a well defined map from $\underline{K}_{H}(\underline{a}, \underline{b})$ to $\underline{K}_{H}(\underline{a}, \underline{b})$ for all $m$ coprime to the exponent of $H$. Therefore, for irreducible characters $\chi \in \operatorname{Irr}\left(C_{G}(\underline{a})\right)$ and $\chi^{\prime} \in \operatorname{Irr}\left(C_{G}(\underline{b})\right)$, if the summand $\chi\left(g \underline{b} g^{-1}\right) \chi^{\prime}\left(g^{-1} \underline{a} g\right)$ appears in formula (3), then all its Galois conjugate also appear. Reordering the sum in orbits under Galois conjugation then yields a sum of rational numbers.

(Notice that since the $\varphi_{a, b}^{(m)}$ are bijections, we may assume $\varphi_{a, b}^{(m)}=\left(\varphi_{a, b}^{\left(m^{-1}\right)}\right)^{-1}$ and hence by the action of the Galois group we obtain indeed orbits.)

Proposition 6.8 For $H=S_{3}, N$ arbitrary or $H=S_{4}, N=Z_{2} \times Z_{2}$, the maps $\varphi_{a, b}^{(m)}$ of proposition 6.7 exist. So the $S$-matrix of the quantum double of the subdirect product is rational.

Proof A solution for the equations given in proposition 6.7 can be computed by a recursion on a computer.

Remark 6.9 The maps $\varphi_{a, b}^{(m)}$ of proposition 6.7 also exist in the cases $H=D_{4}, H=$ $Q_{8}$ and $N \unlhd H$ arbitrary.

\section{Open questions}

The analysis of integral modular data reveals some difficulties which we will certainly also encounter in the case of arbitrary modular data. Therefore it could be interesting to answer the following questions (some of them could be easy to answer): 
(1) Is there an upper bound for the largest norm of an integral modular datum in a given dimension?

(2) What are the integral Fourier matrices with 'small' structure constants (for example in $\{-1,0,1\})$ ?

(3) Are there integral Fourier matrices with the same degrees, nonnegative structure constants and non isomorphic fusion algebras (see example 2)?

(4) Is there an integral Fourier matrix with a unique norm, negative structure constants and an entry unequal to \pm 1 ?

(5) Does an integral Fourier matrix $s$ exist with a column $k$ such that $\forall i s_{i k} \neq 0$ and $\exists i s_{i k} \neq \pm 1$ ?

(6) What are the degrees of congruence multiplication tables?

(7) Is there an integral Fourier matrix with only one degree equal to 1 and of dimension greater than 1 ?

(8) Does the integrality of the structure constants of a congruence multiplication table already follow from the other axioms?

(9) What is the 'modulus' (or the corresponding ideal) in the case of arbitrary modular data?

(10) Are there modular data with rational $S$ and $T$ matrices which do not belong to the infinite sequence of proposition 5.1 (probably yes)?

(11) Classify integral finite group modular data.

\section{Appendix}

A.1 Computation of all integral Fourier matrices up to dimension 12

\section{A.1.1 The norms}

The first step is to list all possible norms. For a given dimension $n>1$, we have to compute all tuples $d_{1} \geq \ldots \geq d_{n}$ with the properties

$$
d_{j} \mid d_{1}, \quad \sqrt{\frac{d_{1}}{d_{j}}} \in \mathbb{Z}, \quad a:=\sum_{i} \frac{1}{d_{i}}=1
$$

for all $1 \leq j \leq n$. For this, we start with $2 \leq d_{n} \leq n$.

By lemma 3.7, if $n$ is odd, we may assume that all $d_{i}$ are squares. If $n$ is even, then the square free part of all $d_{i}$ is the same, so in this case we divide $d_{n}$ by its square free part $p$ and start again in dimension $n-1$ and the above sum now sums up to $a:=p-\frac{p}{d_{n}}$; we replace $d_{n}$ by $\frac{d_{n}}{p}$ which is a square.

Then we start a recursion. There are two lower bounds for the next $d_{i}$ in each step given by the above number $a$ (from which we subtract the inverse of the last $d_{i}$ in each step) and by $d_{i+1}$. The upper bound is $\frac{n}{a}$.

Solutions $d_{1}, \ldots, d_{n}$ to the equation $\frac{1}{d_{1}}+\ldots+\frac{1}{d_{n}}=1$ are called Egyptian fractions. For a given $n$, the $d_{i}$ are bounded by the $n$-th term of Sylvester's sequence: $a_{n+1}=a_{n}^{2}-a_{n}+1, a_{1}=2$ ([15] gives the reference [14]). This information is not useful for the computation because the numbers $a_{n}$ are very large. 


\section{A.1.2 Finding the matrices to a given norm vector}

The second step is much more complicated, because there is a lot of information to exploit in the algorithm. Again, we construct the matrix in a recursive way, row by row. Because it is symmetric, we still have to compute a square matrix after each step.

There are two important observations: it is very useful to test if the structure constants are integers after each step (only for those structure constants which may already be computed of course). Also, if there is a norm with high multiplicity, say $m$, then the recursion will compute almost all permutations of the correcponding block, so approximatively $m$ ! matrices. So it is crucial to keep only representatives of the matrices up to simultaneous permutations of rows and columns before the next recursion step. It is also possible to include several bounds into the computation.

Finding all matrices up to dimension 11 takes just a few minutes, whereas dimension 12 takes about a week on an ordinary PC.

Finding congruence multiplication tables is of course much easier, but you still need to compute all norm vectors for one dimension. This begins to become difficult at dimension 13 , even when using the information of the above propositions.

\section{A.1.3 Finding $T$-matrices}

For a given $s$-matrix $s$, a $T$-matrix

$$
T=\operatorname{diag}\left(t_{1}, \ldots, t_{n}\right)
$$

is a solution of the equations

$$
\sum_{k} t_{i} t_{j} t_{k} \frac{s_{i k} s_{k j}}{\sqrt{d_{k}}}=s_{i j}
$$

for all $1 \leq i, j \leq n$. If $T$ has finite order, then $t_{1}, \ldots, t_{n}$ are roots of unity. In particular, for $i=j=1$ we get $\sum_{k} \frac{\sqrt{d_{1}}}{d_{k}} t_{1}^{2} t_{k}=1$. And if $s_{i j}=0$ for some $1 \leq i, j \leq n$, then $\sum_{k} s_{i k} s_{j k} t_{k}=0$.

If $s$ has small dimension, then there is no need to elaborate a complicated algorithm: it suffices to compute the Gröbner basis of the system of equations (4) in an adequate field. All matrices of the appendix are small enough for this naive method.

\section{A.2 An integral modular datum in dimension 16}

The following matrix is the $S$-matrix of an integral modular datum. A corresponding $T$-matrix does not have finite order, so it is not a modular datum in the sense of Gannon. But the structure constants are nonnegative. Remark also, that it is a congruence 
multiplication table.

$$
\left(\begin{array}{cccccccccccccccc}
1 & 1 & 2 & 6 & 6 & 6 & 6 & 6 & 6 & 6 & 6 & 14 & 14 & 14 & 21 & 21 \\
1 & 1 & 2 & 6 & 6 & 6 & 6 & 6 & 6 & 6 & 6 & 14 & 14 & 14 & -21 & -21 \\
1 & 1 & 2 & 6 & 6 & 6 & 6 & 6 & 6 & 6 & 6 & -7 & -7 & -7 & 0 & 0 \\
1 & 1 & 2 & 6 & -1 & -1 & -1 & -1 & -1 & -1 & -1 & 0 & 0 & 0 & 0 & 0 \\
1 & 1 & 2 & -1 & 6 & -1 & -1 & -1 & -1 & -1 & -1 & 0 & 0 & 0 & 0 & 0 \\
1 & 1 & 2 & -1 & -1 & 6 & -1 & -1 & -1 & -1 & -1 & 0 & 0 & 0 & 0 & 0 \\
1 & 1 & 2 & -1 & -1 & -1 & 6 & -1 & -1 & -1 & -1 & 0 & 0 & 0 & 0 & 0 \\
1 & 1 & 2 & -1 & -1 & -1 & -1 & 6 & -1 & -1 & -1 & 0 & 0 & 0 & 0 & 0 \\
1 & 1 & 2 & -1 & -1 & -1 & -1 & -1 & 6 & -1 & -1 & 0 & 0 & 0 & 0 & 0 \\
1 & 1 & 2 & -1 & -1 & -1 & -1 & -1 & -1 & -1 & 6 & 0 & 0 & 0 & 0 & 0 \\
1 & 1 & 2 & -1 & -1 & -1 & -1 & -1 & -1 & 6 & -1 & 0 & 0 & 0 & 0 & 0 \\
1 & 1 & -1 & 0 & 0 & 0 & 0 & 0 & 0 & 0 & 0 & 2 & -1 & -1 & 0 & 0 \\
1 & 1 & -1 & 0 & 0 & 0 & 0 & 0 & 0 & 0 & 0 & -1 & -1 & 2 & 0 & 0 \\
1 & 1 & -1 & 0 & 0 & 0 & 0 & 0 & 0 & 0 & 0 & -1 & 2 & -1 & 0 & 0 \\
1 & -1 & 0 & 0 & 0 & 0 & 0 & 0 & 0 & 0 & 0 & 0 & 0 & 0 & -1 & 1 \\
1 & -1 & 0 & 0 & 0 & 0 & 0 & 0 & 0 & 0 & 0 & 0 & 0 & 0 & 1 & -1
\end{array}\right)
$$

A solution for a $T$-matrix is for example

$$
T:=\operatorname{diag}\left(1,1,1,1,1,1,1,1,1, \alpha, \alpha^{-1}, 1, \zeta, \zeta^{2}, 1,-1\right),
$$

where $\alpha$ is a root of $X^{2}+5 X+1$ and $\zeta$ a third root of unity.

\section{A.3 A 'rational' modular datum in dimension 18}

The following matrix is the $S$-matrix of an integral modular datum. All corresponding $T$-matrices have finite order and the structure constants are nonnegative, so it is a modular datum in the sense of Gannon. It is not a congruence multiplication table.

$$
\left(\begin{array}{cccccccccccccccccc}
1 & 1 & 1 & 1 & 3 & 3 & 3 & 3 & 3 & 3 & 3 & 3 & 3 & 3 & 3 & 3 & 4 & 4 \\
1 & 1 & 1 & 1 & 3 & 3 & 3 & 3 & -3 & -3 & -3 & -3 & -3 & -3 & -3 & -3 & 4 & 4 \\
1 & 1 & 1 & 1 & -3 & -3 & -3 & -3 & 3 & 3 & 3 & 3 & -3 & -3 & -3 & -3 & 4 & 4 \\
1 & 1 & 1 & 1 & -3 & -3 & -3 & -3 & -3 & -3 & -3 & -3 & 3 & 3 & 3 & 3 & 4 & 4 \\
1 & 1 & -1 & -1 & -1 & 1 & 1 & -1 & 1 & 1 & -1 & -1 & 1 & 1 & -1 & -1 & 0 & 0 \\
1 & 1 & -1 & -1 & 1 & -1 & -1 & 1 & 1 & 1 & -1 & -1 & -1 & -1 & 1 & 1 & 0 & 0 \\
1 & 1 & -1 & -1 & 1 & -1 & -1 & 1 & -1 & -1 & 1 & 1 & 1 & 1 & -1 & -1 & 0 & 0 \\
1 & 1 & -1 & -1 & -1 & 1 & 1 & -1 & -1 & -1 & 1 & 1 & -1 & -1 & 1 & 1 & 0 & 0 \\
1 & -1 & 1 & -1 & 1 & 1 & -1 & -1 & -1 & 1 & 1 & -1 & 1 & -1 & 1 & -1 & 0 & 0 \\
1 & -1 & 1 & -1 & 1 & 1 & -1 & -1 & 1 & -1 & -1 & 1 & -1 & 1 & -1 & 1 & 0 & 0 \\
1 & -1 & 1 & -1 & -1 & -1 & 1 & 1 & 1 & -1 & -1 & 1 & 1 & -1 & 1 & -1 & 0 & 0 \\
1 & -1 & 1 & -1 & -1 & -1 & 1 & 1 & -1 & 1 & 1 & -1 & -1 & 1 & -1 & 1 & 0 & 0 \\
1 & -1 & -1 & 1 & 1 & -1 & 1 & -1 & 1 & -1 & 1 & -1 & -1 & 1 & 1 & -1 & 0 & 0 \\
1 & -1 & -1 & 1 & 1 & -1 & 1 & -1 & -1 & 1 & -1 & 1 & 1 & -1 & -1 & 1 & 0 & 0 \\
1 & -1 & -1 & 1 & -1 & 1 & -1 & 1 & 1 & -1 & 1 & -1 & 1 & -1 & -1 & 1 & 0 & 0 \\
1 & -1 & -1 & 1 & -1 & 1 & -1 & 1 & -1 & 1 & -1 & 1 & -1 & 1 & 1 & -1 & 0 & 0 \\
1 & 1 & 1 & 1 & 0 & 0 & 0 & 0 & 0 & 0 & 0 & 0 & 0 & 0 & 0 & 0 & 1 & -2 \\
1 & 1 & 1 & 1 & 0 & 0 & 0 & 0 & 0 & 0 & 0 & 0 & 0 & 0 & 0 & 0 & -2 & 1
\end{array}\right)
$$

A solution for a $T$-matrix is for example

$$
T:=\operatorname{diag}\left(-1,-1,-1,-1,-1,1,1,-1,-1,1,1,-1,1,-1,-1,1, \zeta^{-1}, \zeta\right),
$$


where $\zeta$ a sixth root of unity. The group spanned by $S$ and $T$ is $\operatorname{PSL}_{2}(\mathbb{Z} / 6 \mathbb{Z})$. Permuting columns of $S$ yields another Fourier matrix which has $T$-matrices such that the groups spanned by $S$ and $T$ are isomorphic to $\mathrm{SL}_{2}(\mathbb{Z} / 12 \mathbb{Z}) / N$ where $N$ is a normal subgroup of order 4 . Remark that these groups are those that appear for the fusion algebra corresponding to the quantum double of $S_{3}$.

As mentioned in the introduction, this modular data is similar to one of the modular data corresponding to the exceptional complex reflection group $G_{8}$. Section 8.2.3 of [3] describes a construction for its fusion algebra as a factor ring of the quantum double of the 9-th group of order 36 in the numbering of GAP.

\section{A.4 Dimension 1 to 12}

This is a list of all isomorphism classes of matrices $s$ satisfying the axioms (1), (2), (3), (4) of definition 3.1 up to dimension 12.

A permutation $\sigma$ as an index means that it is a non isomorphic matrix obtained by permuting the columns of this matrix by $\sigma$. If there are two permutations, then the first one is for the columns, the second one for the rows.

The exponent +6 stands for the construction of proposition 4.5 .

$$
\begin{aligned}
& s(2,1):=\left(\begin{array}{cc}
1 & 1 \\
1 & -1
\end{array}\right), \quad s(4,1):=\left(\begin{array}{cccc}
1 & 1 & 1 & 1 \\
1 & 1 & -1 & -1 \\
1 & -1 & 1 & -1 \\
1 & -1 & -1 & 1
\end{array}\right), \\
& s(4,1)_{(3,4)}, \quad s(7,1):=(1)^{+6}
\end{aligned}
$$

$$
s(8,1):=s(2,1)^{\otimes 3}, \quad s(8,2):=\left(\begin{array}{cccccccc}
1 & 1 & 2 & 2 & 2 & 2 & 3 & 3 \\
1 & 1 & 2 & 2 & 2 & 2 & -3 & -3 \\
1 & 1 & -1 & -1 & -1 & 2 & 0 & 0 \\
1 & 1 & -1 & -1 & 2 & -1 & 0 & 0 \\
1 & 1 & -1 & 2 & -1 & -1 & 0 & 0 \\
1 & 1 & 2 & -1 & -1 & -1 & 0 & 0 \\
1 & -1 & 0 & 0 & 0 & 0 & 1 & -1 \\
1 & -1 & 0 & 0 & 0 & 0 & -1 & 1
\end{array}\right)
$$

$$
s(8,2)_{(7,8)}, \quad s(8,2)_{(4,5)}, \quad s(8,2)_{(4,5)(7,8)}, \quad s(8,2)_{(3,6)(4,5)}, \quad s(8,2)_{(3,6)(4,5)(7,8)}
$$

$$
s(9,1):=\left(\begin{array}{ccc}
1 & 2 & 2 \\
1 & \frac{1}{2} & -1 \\
1 & -1 & \frac{1}{2}
\end{array}\right)^{+6}, \quad s(9,1)_{(5,6),}, \quad s(9,2):=\left(\begin{array}{ccc}
1 & \frac{1}{2} & 1 \\
1 & 2 & -2 \\
1 & -1 & -\frac{1}{2}
\end{array}\right)^{+6}
$$




$$
\begin{aligned}
& s(10,1):=\left(\begin{array}{cccccccccc}
1 & 1 & 1 & 1 & 1 & 2 & 3 & 3 & 3 & 6 \\
1 & 1 & 1 & 1 & 1 & 2 & 3 & 3 & 3 & -6 \\
1 & 1 & 1 & 1 & -5 & -4 & 3 & 3 & -3 & 0 \\
1 & 1 & 1 & -5 & 1 & -4 & 3 & -3 & 3 & 0 \\
1 & 1 & -5 & 1 & 1 & -4 & -3 & 3 & 3 & 0 \\
1 & 1 & -2 & -2 & -2 & 2 & 0 & 0 & 0 & 0 \\
1 & 1 & 1 & 1 & -1 & 0 & -1 & -1 & 1 & 0 \\
1 & 1 & 1 & -1 & 1 & 0 & -1 & 1 & -1 & 0 \\
1 & 1 & -1 & 1 & 1 & 0 & 1 & -1 & -1 & 0 \\
1 & -1 & 0 & 0 & 0 & 0 & 0 & 0 & 0 & 0
\end{array}\right), \\
& s(10,1)_{(7,9),(3,5),} \quad s(10,2):=s(4,1)^{+6}, \quad s(10,2)_{(6,7)} \\
& s(10,3):=\left(\begin{array}{cccccccccc}
1 & 1 & 2 & 3 & 3 & 4 & 4 & 4 & 6 & 6 \\
1 & 1 & 2 & 3 & 3 & 4 & 4 & 4 & -6 & -6 \\
1 & 1 & 2 & 3 & 3 & -2 & -2 & -2 & 0 & 0 \\
1 & 1 & 2 & -1 & -1 & 0 & 0 & 0 & 2 & -2 \\
1 & 1 & 2 & -1 & -1 & 0 & 0 & 0 & -2 & 2 \\
1 & 1 & -1 & 0 & 0 & 1 & 1 & -2 & 0 & 0 \\
1 & 1 & -1 & 0 & 0 & 1 & -2 & 1 & 0 & 0 \\
1 & 1 & -1 & 0 & 0 & -2 & 1 & 1 & 0 & 0 \\
1 & -1 & 0 & 1 & -1 & 0 & 0 & 0 & 0 & 0 \\
1 & -1 & 0 & -1 & 1 & 0 & 0 & 0 & 0 & 0
\end{array}\right), \quad s(10,3)_{(6,8)}
\end{aligned}
$$$$
\begin{aligned}
& s(11,1):=\left(\begin{array}{ccccc}
1 & 2 & 2 & 6 & 6 \\
1 & 2 & 2 & \frac{3}{2} & -3 \\
1 & 2 & 2 & -3 & \frac{3}{2} \\
1 & \frac{1}{2} & -1 & 0 & 0 \\
1 & -1 & \frac{1}{2} & 0 & 0
\end{array}\right)^{+6}, \\
& s(11,2):=\left(\begin{array}{ccccc}
1 & 1 & 3 & 3 & 4 \\
1 & 1 & -3 & -3 & 4 \\
1 & -1 & 1 & -1 & 0 \\
1 & -1 & -1 & 1 & 0 \\
1 & 1 & 0 & 0 & -\frac{1}{2}
\end{array}\right)^{+6}
\end{aligned}
$$$$
s(11,2)_{(6,7),} \quad s(12,1):=\left(\begin{array}{cccccc}
1 & 1 & 4 & 9 & 9 & 12 \\
1 & 1 & 4 & -9 & -9 & 12 \\
1 & 1 & 4 & 0 & 0 & -\frac{3}{2} \\
1 & -1 & 0 & 1 & -1 & 0 \\
1 & -1 & 0 & -1 & 1 & 0 \\
1 & 1 & -\frac{1}{2} & 0 & 0 & 0
\end{array}\right)^{+6} \quad, \quad s(12,1)_{(7,8)}
$$ 


$$
s(12,2):=\left(\begin{array}{cccccccccccc}
1 & 1 & 2 & 2 & 2 & 2 & 6 & 6 & 6 & 6 & 9 & 9 \\
1 & 1 & 2 & 2 & 2 & 2 & 6 & 6 & 6 & 6 & -9 & -9 \\
1 & 1 & 2 & 2 & 2 & 2 & -3 & -3 & -3 & 6 & 0 & 0 \\
1 & 1 & 2 & 2 & 2 & 2 & -3 & -3 & 6 & -3 & 0 & 0 \\
1 & 1 & 2 & 2 & 2 & 2 & -3 & 6 & -3 & -3 & 0 & 0 \\
1 & 1 & 2 & 2 & 2 & 2 & 6 & -3 & -3 & -3 & 0 & 0 \\
1 & 1 & -1 & -1 & -1 & 2 & 0 & 0 & 0 & 0 & 0 & 0 \\
1 & 1 & -1 & -1 & 2 & -1 & 0 & 0 & 0 & 0 & 0 & 0 \\
1 & 1 & -1 & 2 & -1 & -1 & 0 & 0 & 0 & 0 & 0 & 0 \\
1 & 1 & 2 & -1 & -1 & -1 & 0 & 0 & 0 & 0 & 0 & 0 \\
1 & -1 & 0 & 0 & 0 & 0 & 0 & 0 & 0 & 0 & 1 & -1 \\
1 & -1 & 0 & 0 & 0 & 0 & 0 & 0 & 0 & 0 & -1 & 1
\end{array}\right),
$$

$s(12,2)_{(11,12)}$

$$
s(12,3):=\left(\begin{array}{cccccccccccc}
1 & 1 & 2 & 3 & 3 & 6 & 6 & 8 & 8 & 8 & 12 & 12 \\
1 & 1 & 2 & 3 & 3 & 6 & 6 & 8 & 8 & 8 & -12 & -12 \\
1 & 1 & 2 & 3 & 3 & 6 & 6 & -4 & -4 & -4 & 0 & 0 \\
1 & 1 & 2 & 3 & 3 & -2 & -2 & 0 & 0 & 0 & 4 & -4 \\
1 & 1 & 2 & 3 & 3 & -2 & -2 & 0 & 0 & 0 & -4 & 4 \\
1 & 1 & 2 & -1 & -1 & 2 & -2 & 0 & 0 & 0 & 0 & 0 \\
1 & 1 & 2 & -1 & -1 & -2 & 2 & 0 & 0 & 0 & 0 & 0 \\
1 & 1 & -1 & 0 & 0 & 0 & 0 & -1 & -1 & 2 & 0 & 0 \\
1 & 1 & -1 & 0 & 0 & 0 & 0 & -1 & 2 & -1 & 0 & 0 \\
1 & 1 & -1 & 0 & 0 & 0 & 0 & 2 & -1 & -1 & 0 & 0 \\
1 & -1 & 0 & 1 & -1 & 0 & 0 & 0 & 0 & 0 & 0 & 0 \\
1 & -1 & 0 & -1 & 1 & 0 & 0 & 0 & 0 & 0 & 0 & 0
\end{array}\right)
$$

$s(12,3)_{(8,10)}, \quad s(12,3)_{(6,7)}, \quad s(12,3)_{(6,7)(8,10)}$

$$
s(12,4):=\left(\begin{array}{cccccccccccc}
1 & 1 & 2 & 6 & 6 & 6 & 6 & 10 & 10 & 10 & 15 & 15 \\
1 & 1 & 2 & 6 & 6 & 6 & 6 & 10 & 10 & 10 & -15 & -15 \\
1 & 1 & 2 & 6 & 6 & 6 & 6 & -5 & -5 & -5 & 0 & 0 \\
1 & 1 & 2 & 1 & 1 & 1 & -4 & 0 & 0 & 0 & 0 & 0 \\
1 & 1 & 2 & 1 & 1 & -4 & 1 & 0 & 0 & 0 & 0 & 0 \\
1 & 1 & 2 & 1 & -4 & 1 & 1 & 0 & 0 & 0 & 0 & 0 \\
1 & 1 & 2 & -4 & 1 & 1 & 1 & 0 & 0 & 0 & 0 & 0 \\
1 & 1 & -1 & 0 & 0 & 0 & 0 & 1 & 1 & -2 & 0 & 0 \\
1 & 1 & -1 & 0 & 0 & 0 & 0 & 1 & -2 & 1 & 0 & 0 \\
1 & 1 & -1 & 0 & 0 & 0 & 0 & -2 & 1 & 1 & 0 & 0 \\
1 & -1 & 0 & 0 & 0 & 0 & 0 & 0 & 0 & 0 & 1 & -1 \\
1 & -1 & 0 & 0 & 0 & 0 & 0 & 0 & 0 & 0 & -1 & 1
\end{array}\right)
$$

$s(12,4)_{(11,12)}, \quad s(12,4)_{(8,10)}, \quad s(12,4)_{(8,10)(11,12)}, \quad s(12,4)_{(5,6)}$ 
$s(12,4)_{(5,6)(11,12)}, \quad s(12,4)_{(5,6)(8,10)}, \quad s(12,4)_{(5,6)(8,10)(11,12),} s(12,4)_{(4,7)(5,6)}$

$$
\begin{aligned}
& s(12,4)_{(4,7)(5,6)(11,12),} s(12,4)_{(4,7)(5,6)(8,10),} \quad s(12,4)_{(4,7)(5,6)(8,10)(11,12)} \\
& s(12,5):=\left(\begin{array}{cccccc}
1 & \frac{1}{2} & \frac{1}{2} & \frac{1}{2} & \frac{1}{2} & \frac{1}{2} \\
1 & -1 & -1 & -1 & -1 & 2 \\
1 & -1 & -1 & -1 & 2 & -1 \\
1 & -1 & -1 & 2 & -1 & -1 \\
1 & -1 & 2 & -1 & -1 & -1 \\
1 & 2 & -1 & -1 & -1 & -1
\end{array}\right)^{+6} \quad, \quad s(12,5)_{(6,8)}, \quad s(12,5)_{(5,9)(6,8)} \\
& s(12,6):=\left(\begin{array}{cccccc}
1 & 1 & 1 & 1 & 1 & 2 \\
1 & 1 & 1 & 1 & -2 & -1 \\
1 & 1 & 1 & -2 & 1 & -1 \\
1 & 1 & -2 & 1 & 1 & -1 \\
1 & -2 & 1 & 1 & 1 & -1 \\
1 & -\frac{1}{2} & -\frac{1}{2} & -\frac{1}{2} & -\frac{1}{2} & \frac{1}{2}
\end{array}\right)^{+6}, \quad s(12,6)_{(6,7),} s(12,6)(5,8)(6,7) \\
& s(12,7):=\left(\begin{array}{cccccc}
1 & \frac{1}{2} & 1 & \frac{3}{2} & \frac{3}{2} & \frac{3}{2} \\
1 & \frac{7}{2} & 4 & -\frac{3}{2} & -\frac{3}{2} & -\frac{3}{2} \\
1 & 2 & -2 & 0 & 0 & 0 \\
1 & -\frac{1}{2} & 0 & \frac{1}{2} & \frac{1}{2} & -\frac{3}{2} \\
1 & -\frac{1}{2} & 0 & \frac{1}{2} & -\frac{3}{2} & \frac{1}{2} \\
1 & -\frac{1}{2} & 0 & -\frac{3}{2} & \frac{1}{2} & \frac{1}{2}
\end{array}\right)^{+6}, \quad s(12,7)_{(7,9)}
\end{aligned}
$$

Acknowledgements The author thanks G. Malle for his support and many helpful suggestions. He is also very grateful to T. Gannon and the referee who have contributed Proposition 6.1 and to the referee for Proposition 5.3.

\section{References}

1. Bakalov, B., Kirillov, A. Jr.: Lectures on Tensor Categories and Modular Functors. University Lecture Series, vol. 21. American Mathematical Society, Providence (2001)

2. Coste, A., Gannon, T., Ruelle, P.: Finite group modular data. Nucl. Phys. B 581(3), 679-717 (2000)

3. Cuntz, M.: Fourier-Matrizen und Ringe mit Basis. Dissertation, Universität Kassel (2005)

4. Dijkgraaf, R., Vafa, C., Verlinde, E., Verlinde, H.: The operator algebra of orbifold models. Commun. Math. Phys. 123(3), 485-526 (1989)

5. Gannon, T.: The automorphisms of affine fusion rings. Adv. Math. 165(2), 165-193 (2002)

6. Gannon, T.: Modular data: the algebraic combinatorics of conformal field theory. J. Algebraic Combin. 22(2), 211-250 (2005)

7. Geck, M., Hiss, G., Lübeck, F., Malle, G., Michel, J., Pfeiffer, G.: CHEVIE—a system for computing and processing generic character tables. http://www.math.jussieu.fr/ jmichel/chevie/chevie.html (2006)

8. Geck, M., Malle, G.: Fourier transforms and Frobenius eigenvalues for finite Coxeter groups. J. Algebra 260(1), 162-193 (2003)

9. Lusztig, G.: Leading coefficients of character values of Hecke algebras. In: The Arcata Conference on Representations of Finite Groups (Arcata Calif., 1986). Proc. Sympos. Pure Math., vol. 47, pp. 235262. Amer. Math. Soc., Providence (1987)

10. Lusztig, G.: Exotic Fourier transform. Duke Math. J. 73(1), 227-241, 243-248 (1994). With an appendix by Gunter Malle 
11. Malle, G.: Unipotente Grade imprimitiver komplexer Spiegelungsgruppen. J. Algebra 177(3), 768826 (1995)

12. Malle, G.: Spetses. In: Proceedings of the International Congress of Mathematicians, vol. II (Berlin, 1998). Extra Vol. II, pp. 87-96 (1998) (elektronisch)

13. Shephard, G.C., Todd, J.A.: Finite unitary reflection groups. Can. J. Math. 6, 274-304 (1954)

14. Singmaster, D.: The number of representations of one as a sum of unit fractions. Unpublished manuscript (1972)

15. Sloane, N.J.A.: The on-line encyclopedia of integer sequences. Published electronically at http://www.research.att.com/ njas/sequences/ (2006) 